\title{
Quintus Smyrnaeus und die Tradition des epischen Musenanrufs*
}

\author{
SILVIO BÄR
}

\section{Die Gattungstradition des epischen Musenanrufs: Initialproömium und Binnenproömium ${ }^{1}$}

Seit Ilias und Odyssee und somit seit Anbeginn der griechischen Literatur gehört es zum festen Bestandteil eines Epos, dass dieses vom epischen ,Ich“ mit einem kurzen Abschnitt eingeleitet wird, in welchem einerseits eine Göttin bzw. Muse oder auch kollektiv die Musen angerufen und um Inspiration gebeten werden und andererseits die anschliessende Erzählung thematisch umrissen wird. ${ }^{2}$ Während in den homerischen Epen die Beziehung zwischen Dichter und Muse(n) grundsätzlich vorausgesetzt wird $^{3}$ und das epische ,Ich“ anonym bleibt, tritt mit Hesiod erstmals ein Dichter namentlich ${ }^{4}$ und somit als Individuum in Erscheinung und verwandelt mit seiner Musenweihe ${ }^{5}$ das statische Verhältnis zwischen Dichter

* Herrn Prof. Dr. Christoph Riedweg (Zürich / Rom), Herrn Prof. Dr. Manuel Baumbach (Zürich), Herrn Prof. Ewen L. Bowie (Corpus Christi College, Oxford) und Frau lic. phil. Nicola Dümmler (Zürich) sowie allen Teilnehmern der Quintus-Smyrnaeus-Tagung sei für die kritische Lektüre dieses Aufsatzes und für vielfältige Anregungen und Verbesserungsvorschläge herzlich gedankt. Zugrunde liegende Textausgaben für Zitate sind: Quintus Smyrnaeus: Pompella (2002); Homer: van Thiel (1991) und (1996); Hesiod: Solmsen / Merkelbach / West ( $\left.{ }^{3} 1990\right)$; Kallimachos: Asper (2004). Sämtliche Übersetzungen griechischer und lateinischer Textpassagen stammen von mir, sofern ad loc. nicht anders vermerkt.

1 Die nachstehenden Ausführungen stützen sich im Wesentlichen auf: Otto (1954); Klotz (1965); Koster (1970); Lenz (1980); Pötscher (1986); Race (1992); Mandelartz (1993); Schmitzer (2000); Gärtner (2001).

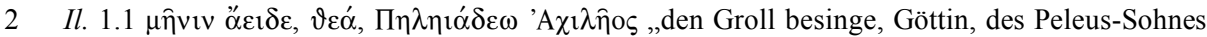

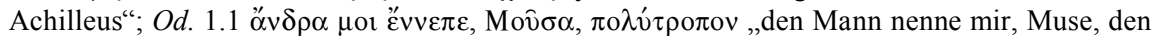

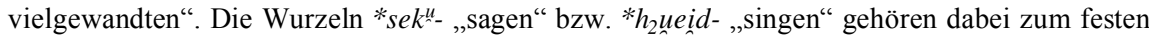
Repertoire.

3 Eine gewisse Ausnahme bildet das Binnenproömium in Il. 2.484-492, welches Ansätze zur Reflexion der sängerischen Tätigkeit zeigt. Zu dieser Stelle vgl. auch Kap. 3.1.

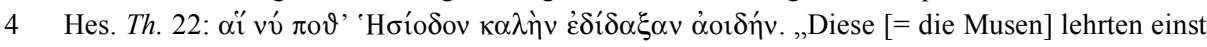
Hesiod die schöne Sangeskunst.“

$5 \quad$ Hes. Th. 22-34. Zu dieser Stelle s.u. Kap. 3.2. 
und Muse(n) in einen dynamischen Prozess. ${ }^{6}$ Die Frage nach der ursprünglichen Funktion der epischen Musenanrufung ${ }^{7}$ soll hier ebenso wenig diskutiert werden wie das Problem des Verhältnisses zwischen Dichter und Muse $(n)^{8}$ - erwähnt sei einzig, dass sowohl die Musen an und für sich als auch die Praxis einer Götteranrufung zum Zwecke dichterischer Inspiration offenbar genuin griechisch sind. ${ }^{9}$ Für unsere Zwecke von Bedeutung ist lediglich die Tatsache, dass - unabhängig von deren ursprünglicher Funktion - ein antikes Epos nach homerischer Gattungstradition mit jenen genannten einleitenden Elementen $\mathrm{zu}$ beginnen hat, wofür in der modernen Forschung zusammenfassend der aus der antiken Rhetorik

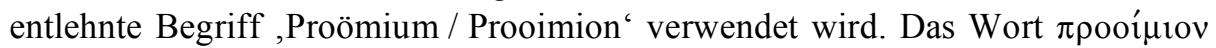
ist bei Pindar zum ersten Mal belegt (N. 2.3) und seit Aristoteles Bestandteil der rhetorischen Theorie. ${ }^{10}$ Proömien, welche nicht zu Beginn, sondern inmitten eines Werks stehen, werden in der Regel differenziert ,Binnenproömien' genannt. ${ }^{11}$

Bei den griechischen und lateinischen Epikern nach Homer und Hesiod ist ein stetiges Bemühen zu erkennen, die genannten proömialen Elemente in origineller Weise immer wieder zu variieren, zu individualisieren und somit jeweils neu zu beleben: ${ }^{12}$ Apollonios Rhodios etwa ordnet die Musen dem ebenfalls für die

6 Vgl. Kambylis (1965) 16: „Homer und die homerischen Sänger sind berufen, Hesiodos (und später Kallimachos und Properz) werden berufen. Wesentlich ist dabei, dass diese Berufung den Dichter einen neuen Weg gehen lässt, und dieser Weg ist ein erstes Kennzeichen des über sich selbst und seine Kunst reflektierenden Künstlers.“

7 Hierbei ist wohl an einen starken ursprünglichen Zusammenhang mit den Produktionsbedingungen der oral poetry und der daraus resultierenden Notwendigkeit des Memorierens und Auswendig-Rezitierens zu denken; vgl. Lenz (1980) 21-26.

8 Die hauptsächliche Frage ist, ob der Dichter nur als Sprachrohr der Muse(n) zu verstehen ist oder ob (und falls ja, wie viel) Eigenständigkeit ihm zugesprochen werden kann. Eine Übersicht über die wichtigsten diesbezüglichen Auffassungen bietet Pötscher (1986).

9 Gemäss West (1997) 170 existiert zwar in der altorientalischen Literatur die Vorstellung von Inspirationsgottheiten, doch sind sowohl Musen o.dgl. als auch so etwas wie ein Musenanruf daselbst unbekannt.

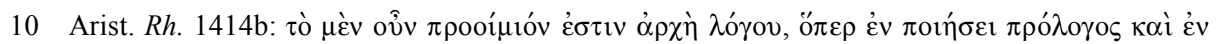

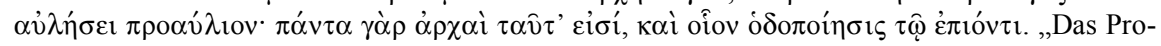
ömium nun ist der Anfang einer Rede, so wie in der Dichtung der Prolog und beim Oboenspiel das Vorspiel. Denn all diese Dinge sind Anfänge und sozusagen eine Wegbereitung für das darauf Folgende." - Es handelt sich also um einen hauptsächlich rhetorischen Terminus, der zwar auch, aber keinesfalls ausschliesslich zur Bezeichnung für die Einleitung von Epen gebraucht wird. Nicht zu vergessen ist ausserdem der Begriff $\pi$ pooífıv als Gattungsbezeichnung für die Homerischen Hymnen (vgl. Thuc. 3.104).

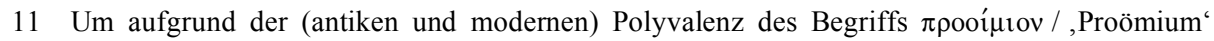
keine Verwirrung aufkommen zu lassen, verwende ich im Folgenden den Ausdruck ,Proömium“ für alle Arten von Proömien, während ich werkeinleitende Proömien als ,Initialproömien` bezeichne.

12 Vgl. dazu Koster (1970) 151-158; Schmitzer (2000). - Die nachstehende Aufzählung bietet nur eine knappe Auswahl an Beispielen zur Veranschaulichung des Sachverhalts. 
Dichtung zuständigen Apollon unter, ${ }^{13}$ während er sich an anderer Stelle an eine einzelne Muse mit Namen wendet. ${ }^{14}$ Arat ruft Zeus an und verknüpft somit die Götteranrufung auf Kosten des Inspirationstopos stärker mit der Themenangabe. ${ }^{15}$ In der lateinischen Dichtung scheint Vergil zu Beginn der Aeneis die Themenangabe vom Inspirationstopos zu lösen, um kurz darauf doch noch einen Musenanruf ,nachzuschieben'. ${ }^{16}$ Ovid schliesslich, das enfant terrible der antiken Hexameterdichtung, parodiert in den Fasti den traditionellen Wahrheitsanspruch des Musenanrufs, indem er drei Musen erfolglos über die Etymologie eines Monatsnamens streiten lässt. ${ }^{17}$ Die Liste der Variationen und Abwandlungen liesse sich fortsetzen.

Bei alledem ist jedoch festzustellen, dass ein Proömium bzw. proömiale Elemente zu Werkbeginn selten völlig aufgegeben werden. Wo dies doch der Fall ist - etwa bei Nikander, Dionysius Periegetes oder dem älteren Oppian -, haben wir es mit nicht-mythischen Erzählungen zu tun, welche rein menschliches Wissen zum Gegenstand haben und deshalb keine göttliche Inspiration benötigen. ${ }^{18}$ Wer sich nun Quintus Smyrnaeus' Posthomerica zur Lektüre vornimmt, wird ein aufgrund der skizzierten epischen Tradition erwartetes Initialproömium vermissen, insbesondere da es sich um eine Fortsetzung der homerischen Ilias und also um ein traditionelles Epos mythischen Inhalts handelt. ${ }^{19}$ Im 12. Buch dagegen, welches von der griechischen List mit dem hölzernen Pferd berichtet, wird man auf ein Binnenproömium stossen, in welchem ein ,Ich'-Sprecher verkündet, er

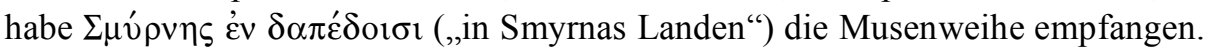
Diese Passage ist der einzige Anhaltspunkt, weshalb Quintus, über dessen Vita wir sonst keinerlei Kenntnis haben, in der Stadt Smyrna lokalisiert und deshalb als Smyrnaeus apostrophiert wird. Ziel der vorliegenden Arbeit ist es, die Tat-

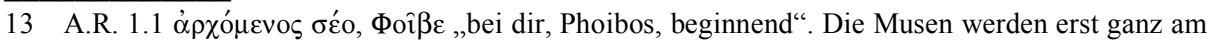

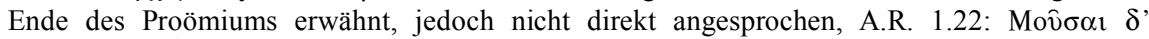

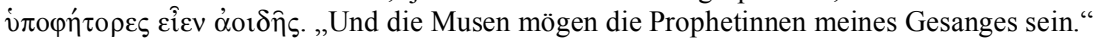

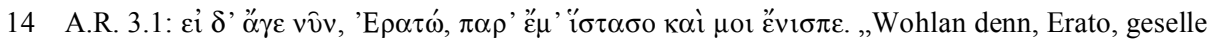
dich nun zu mir und gib mir «den Gesang〉 ein!“

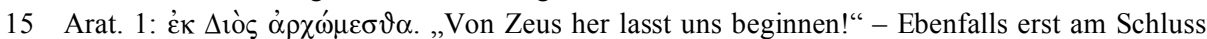
des Proömiums (16-18) werden die Musen dann doch noch angerufen.

16 Verg. Aen. 1.1: arma virumque cano. „Die Waffen und den Mann besinge ich.“ Der Indikativ cano klammert jeglichen göttlichen Beistand o.dgl. völlig aus und rückt das epische ,Ich“ ins alleinige Zentrum. Dieser Traditionsbruch wird jedoch kurz darauf korrigiert, 1.8 Musa, mihi causas memora „Muse, rufe mir die Gründe in Erinnerung“.

17 Ov. Fast. 5.1-110.

18 Vgl. Serv. Aen. 1.8: sane observandum est, ut non in omnibus carminibus numen aliquod invocetur, nisi cum aliquid ultra humanam possibilitatem requirimus. „Es ist freilich zu beachten, dass nicht in allen Gedichten irgendeine Gottheit angerufen werden soll, ausser wenn wir etwas jenseits menschlicher Möglichkeiten suchen.“- Vgl. auch Koster (1970) 153-155.

19 Anders als Quintus verzichtet dessen Zeitgenosse Triphiodor nicht auf den Musenanruf: Er ruft

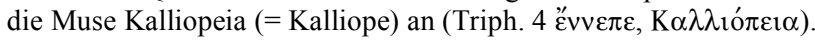


sache des nicht-vorhandenen Proömiums zu Werkbeginn einerseits und den Musenanruf in Q.S. 12.306-313 andererseits zu analysieren, zu interpretieren und auf mögliche Implikationen hin zu befragen. Sodann soll die Frage gestellt werden, ob die Erwähnung von Smyrna als Heimatort des ,Ich'-Sprechers, des impliziten Autors, tatsächlich als autobiographische Aussage des realen Autors Quintus gelten kann. Unter dem Aspekt einer poetologisch-topischen Lesart soll dabei der Versuch einer ganzheitlichen Deutung der beiden Stellen, welche den vermeintlich - autobiographischen Passus integriert, versucht werden.

\title{
2. Das fehlende Initialproömium
}

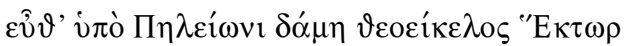

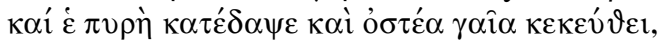

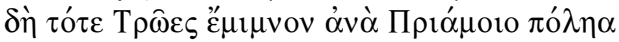

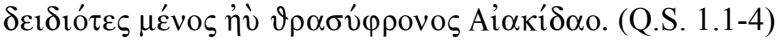 \\ Nachdem der götterähnliche Hektor vom Peleussohn bezwungen ward \\ und der Scheiterhaufen ihn verzehrt hatte und die Erde seine Knochen barg, \\ da blieben die Troer in der Stadt des Priamos, \\ weil sie den ungestümen Zorn des mutig gesinnten Aiakiden fürchteten.
}

Die irritierende Tatsache des fehlenden Initialproömiums zu Beginn der Posthomerica ist in der Forschung unterschiedlich beurteilt worden. Eine traditionelle Auffassung deutet diesen Umstand als Hinweis darauf, dass Quintus ganz einfach ein Epos in unmittelbarem Anschluss an die Ilias verfassen wollte; vgl. z.B. Keydell (1963) 1273: „Qu. wollte ein Epos schreiben, das die Lücke zwischen Ilias und Odyssee ausfüllte; das wird dadurch deutlich, dass er auf ein Prooemium verzichtet hat." Eine modernere Auffassung schreibt den Eingangsversen zwar einen gewissen proömialen Charakter zu, deutet selbigen aber gleichwohl im Zusammenhang eines möglichst direkten, lückenlosen Anschlusses an die Ilias; vgl. z.B. Schenk (1997) 377: „Die Passage hat trotz ihres erzählerischen Charakters die Funktion eines Proömiums, das der Grundlegung des eigenen Ansatzes dient. Der direkte Anschluss an das Ende des 24. Iliasgesanges exponiert die Posthomerica als Fortsetzung des homerischen Epos.“20 Eine völlig gegenteilige Ansicht vertritt dagegen Appel: Fussend auf der spekulativen Ansicht, die Posthomerica stellten eine Kompilation ursprünglich autonomer Gesänge dar und seien erst zu einem späteren Zeitpunkt von Quintus zu einem Gesamtepos zusammengefügt worden (sog. ,Einzelliedtheorie'), glaubt er, unser Dichter habe

20 Ähnlich schon, wenn auch noch vager, Vian (1963) 3: „Préambule [...] servant de transition avec l'Iliade et d'introduction générale.“ 
gar keinen Anschluss an die Ilias bezweckt und genau darum auf ein Proömium verzichtet: „Wenn Quintus an eine unmittelbare Anknüpfung an die Ilias gedacht hätte, wäre es ihm leicht gewesen, das besser zu handhaben. Es hätte genügt, eine kleine Änderung in den vorhandenen Text ei«n`zuführen, um ein solches Ziel gut zu erreichen." 21

Alle diese Ansätze beruhen freilich auf der Prämisse einer monokausalen Erklärung: Entweder habe Quintus den direkten Anschluss an Homer gesucht oder eben nicht. Ich möchte dagegen im Folgenden eine neue Interpretation vorlegen, welche beide Deutungsmuster miteinander in Einklang zu bringen sucht. Zu diesem Zweck werde ich mich den einleitenden 17 Versen der Posthomerica zuerst unter einem inhaltlich-kompositorischen, sodann unter einem poetologischen Gesichtspunkt widmen.

\subsection{Inhalt und Komposition der einleitenden Verse}

Obschon den Posthomerica ein Initialproömium im traditionellen Sinne evidentermassen fehlt, lässt sich die Auffassung, Quintus stürze sich gleich mit dem ersten Satz in medias res und schliesse in der Erzählung der Geschehnisse unmittelbar an das Ende der Ilias an, ${ }^{22}$ in dieser Form nicht aufrechterhalten. Genau genommen beginnt nämlich die Fortsetzung der Ilias erst mit Vers 18, während die Verse 1-17 noch ganz dem iliadischen Geschehen verpflichtet sind: Auf die zitierten Verse 1-4, welche eine allgemeine thematische Situierung mit Worten zur troischen Befindlichkeit verbinden, folgt ein Gleichnis, in dem die Troer mit flüchtenden Rindern und Hektor mit einem wütenden Löwen verglichen werden (5-8). Die folgenden Verse (9-14), angeschlossen mittels eines participium coniunctum (9: $\mu \nu \eta \sigma \alpha ́ \alpha \varepsilon v o l)$, rekapitulieren gedanklich (d.h. in der Erinnerung der Trojaner) Achilleus' grausames Wüten am Idagebirge, am Fluss Skamander und auf dem Meer sowie Hektors Tötung und Schleifung. Die abschliessenden drei Verse (15-17) führen den Kausalzusammenhang zwischen der Furcht der Trojaner und ihrem Verbleiben innerhalb der Mauern Ilions noch einmal vor Augen und bieten gleichzeitig einen kurzen Ausblick auf das $\tau \dot{\varepsilon} \lambda \lambda_{\diamond}$ des ganzen Epos: die unabwendbare Zerstörung Trojas. Insbesondere der die ,Einleitung ' abschliessende Vers 17 (mit dem Städtenamen Tpoíns prägnant am Versschluss) deutet das tragische Ende des Krieges bereits voraus. Auf sprachlicher Ebene werden zentrale Schlüsselbegriffe - der bezwungene Hektor, das verzehrende Feuer, das Ver-

21 Appel (1994c) 6. - Eine kritische Auseinandersetzung mit Appels Thesen bietet Schenk (1997) 365 Anm. 5 und 377 Anm. 25.

22 So beispielsweise Bates (1931) 2: „The poet loses no time, but plunges at once into his narrative." 
bleiben der Troer in der Stadt sowie ihre quälenden Erinnerungen - innerhalb des Einleitungsteils wiederholt und geben diesem somit einen kompositionellen Rahmen:
$1.1 \delta \alpha \dot{\mu} \mu \eta(. . .)^{\prime \prime} \mathrm{\kappa} \tau \omega \rho$
$1.2 \pi v \rho \eta$

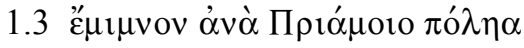
$1.9 \mu \nu \eta \sigma \alpha ́ \mu \varepsilon v o r$

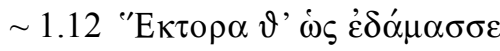
$\sim 1.17 \pi v \rho i ́$

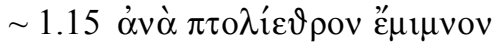
$\sim 1.15 \mu \nu \eta \sigma \vartheta \varepsilon \dot{v} \tau \varepsilon \varsigma$

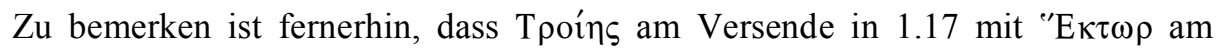
Versende in 1.1 korrespondiert - indem die beiden Namen im ersten bzw. letzten Teil der ,Einleitung' parallelisiert werden, wird Hektors Tod somit symptomatisch für den bevorstehenden Untergang der ganzen Stadt. Das persönliche Schicksal des grössten trojanischen Helden präfiguriert das kollektive Schicksal ganz Trojas, es hat - narratologisch gesprochen - die Funktion einer mise-enabyme. Ähnliches gilt für das Motiv des Feuers in der Einleitung: $\pi v p i ́$ in 1.17 bezieht sich auf die Zerstörung Trojas durch das Feuer, $\pi v \rho \eta ́$ in 1.2 auf den Scheiterhaufen, auf dem Hektor verbrannt wurde. ${ }^{23}$

Inhaltlich stimmen die rekapitulierten Ereignisse (9-14) mit den homerischen Geschehnissen überein - mit einer Ausnahme: Mit der Schleifung Hektors um die Mauern Trojas weicht Quintus von der homerischen Version ab, denn in der Ilias schleift Achilleus die Leiche zuerst zu den Schiffen der Griechen und darauf um Patroklos' Grab. ${ }^{24}$ Quintus hingegen stützt sich auf eine alternative Sagenversion, welche bei Euripides und Vergil schon fassbar ist. ${ }^{25} \mathrm{Ob}$ diese Version auf den Epischen Zyklus zurückgeht, ${ }^{26}$ muss freilich Spekulation bleiben. Jedenfalls wird hier auf der motivischen Ebene deutlich, dass Homer für Quintus zwar das hauptsächliche, jedoch mitnichten das alleinige Vorbild ist und dass er durchaus auch auf andere, alternative Quellen und Versionen zurückgreift, dass er sich also sowohl an die homerischen Epen anlehnt als auch sich gleichzeitig von ebendiesen absetzt. $^{27}$

23 Herrn Prof. Dr. Christoph Riedweg und Herrn Prof. Dr. Manuel Baumbach sei für die zahlreichen wertvollen Hinweise zur Mikrostruktur dieser Verse herzlich gedankt.

24 Vgl. Il. 22.464-465 und 24.17-18.

25 Vgl. Eur. Andr. 107-108; Verg. Aen. 1.483-484 und 2.278-279. Vgl. auch Vian (1963) 12 Anm. 1; James (2004) 269; Gärtner (2005) 42 mit Anm. 8-14. Für ein paar weitere Reflexe dieser Sagenversion vgl. ferner Kopff (1981) 930, der jedoch Q.S. 1.11 nicht nennt. Quintus selber kommt an zwei weiteren Stellen darauf zurück (Q.S. 1.112 und 14.133).

26 So Vian (1963) 12 Anm. 1: ,remonte sans doute au Cycle“. Vgl. auch Kopff (1981) 931.

27 Zu kurz greift m.E. die Erklärung von James (2004) 269, da er die poetologische Implikation von Quintus',Abweichung' nicht berücksichtigt: „Though just a slight variation on the Iliad's version $[\ldots]$ and attested in earlier literature [...], it exemplifies Quintus' readiness to depart from Homeric authority.“ - Zum Motiv der Tötung und Schleifung Hektors in den Posthomeri$c a$ vgl. auch den Beitrag von Knut Usener in diesem Band. 
Die ersten 17 Verse der Posthomerica stellen also, etwas überspitzt formuliert, eine Art ,Ilias in Miniatur-Format' dar, insofern als sie die hauptsächlichen Geschehnisse jenes Epos noch einmal wachrufen: den Zorn des Achilleus und dies der dramatische Höhepunkt der ganzen Ilias-Handlung - sein Wüten und Morden, welches in der Tötung und Schleifung Hektors gipfelt, und die daraus resultierende Katastrophe für die Trojaner. Dabei wird - und darin zeigt sich ein gelungener Kunstgriff des Dichters - die Rekapitulation des iliadischen Geschehens nicht in einer simplen Zusammenfassung vorgenommen, sondern sie wird unter Beibehaltung der erzählten Zeit in das Innere der Figuren, der trojanischen Männer und Frauen, verlegt. Quintus gelingt es somit, denjenigen Punkt auf der Zeitachse, von dem aus er seine eigene Erzählung beginnen und fortsetzen will, $\mathrm{zu}$ fokussieren und gleichzeitig dennoch auf die davor liegenden Ereignisse zurückzugreifen, ohne dass er dabei in der Erzählung als solcher zurückspringen muss. Der Ausgangspunkt der erzählten Zeit ist fixiert, die Rückblende findet von dort aus nur in den Köpfen der beteiligten personae statt. Somit erreicht Quintus zweierlei: Einerseits kann er unmittelbar an das Ende der Ilias anknüpfen und von da aus weiterfahren - die Posthomerica stellen also, so besehen, tatsächlich einen direkten Anschluss an ihr homerisches Vorbild dar. Andererseits kann er die narrative Technik der Rückblende, die ja in Ilias und Odyssee so meisterhaft gehandhabt wird, gleich zu Beginn anwenden und somit nicht zuletzt auch sein Können als ,Homeride' unter Beweis stellen. ${ }^{28}$ Zugleich gelingt es dem Dichter, auf einem Tableau von 17 Versen die momentane Stimmung eindringlich zu schildern sowie den dramatischen Höhepunkt des Vorgängerepos noch einmal deutlich zu evozieren. Somit macht Quintus nicht bloss die vorliegende Situierung des Geschehens unmissverständlich klar, sondern er versetzt den Leser auch in die dafür passende emotionale Lage. Entsprechend wirkungsvoll ist dann der Fortgang des Geschehens, der in Vers 18 einsetzt: Der plötzliche Auftritt der Amazonenkönigin Penthesileia wirkt wie eine Götterepiphanie, ihr unvermutetes Erscheinen in der verzweifelten, ausweglosen Situation wie ein lichter Hoffnungsschimmer am trüben Horizont.

Indem Quintus in den ersten 17 Versen die vergangenen Heldentaten des Achilleus in den Mittelpunkt stellt, wird eine Inhaltsangabe bezüglich des Folgenden, welche für ein Proömium zu erwarten wäre, indirekt dennoch vorgenommen. Im weiteren Verlauf des Epos wird nämlich klar, dass Achilleus und seine Heldentaten bis zu seinem Tod und in einem gewissen Sinne auch noch danach im Mittelpunkt stehen: Nach zwei weiteren grossen Aristien gegen Penthesileia und Memnon (Bücher 1 und 2), welche seine iliadische Aristie gegen Hektor fortsetzen, nach seinem Tod (Buch 3), nach den für ihn veranstalteten

28 Genuin homerisch ist, nebst der hexametrischen Kunstsprache, auch die Gleichnistechnik, von der Quintus in den Versen 5-9 Gebrauch macht. 
Leichenspielen (Buch 4) und nach dem Streit zwischen Odysseus und Aias um seine Waffen (Buch 5) setzt sein Sohn Neoptolemos das Erbe des Vaters im Kampf gegen den letzten bedeutenden Trojanerhelden Eurypylos fort (Bücher 68). ${ }^{29}$ Danach nimmt Neoptolemos' Bedeutung zwar zeitweilig ab (u.a. zugunsten Philoktets in den Büchern 9 und 10), er bleibt aber nach wie vor als wichtiger griechischer Held grundsätzlich präsent. In Buch 14 erscheint sodann Achilleus noch einmal persönlich, und zwar nunmehr (und somit umso wirkungsvoller und theatralischer) als Toter dem Neoptolemos im Traum, um in einem längeren Monolog Polyxenas Opferung zu fordern (Q.S. 14.179-227). ${ }^{30}$ Somit ist also Achilleus (direkt oder indirekt) über weite Teile der Posthomerica sehr präsent und zentral, auch über seinen Tod hinaus.

Des Weiteren ist auf zwei Stellen hinzuweisen, an welchen Achilleus und seine Taten im Werk selber von einem Sänger besungen werden: Im 4. Buch, kurz nach Achilleus' Tod und als Auftakt zu seinen Leichenspielen, preist Nestor

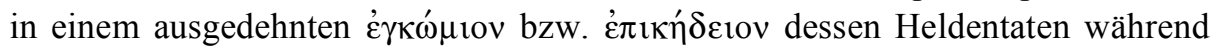
der Belagerungszeit (Eroberungen an der kleinasiatischen Küste) und während des Kriegsgeschehens bis zu seinem Tod; es folgt sodann ein eulogium auf seine Schönheit, seine Stärke und sein sportliches Geschick (Q.S. 4.146-168). ${ }^{31} \mathrm{Im} 14$. Buch schliesslich heisst es, dass die achaiischen Sänger bei den Siegesfestivitäten den ganzen Krieg noch einmal rekapitulieren, wobei naturgemäss dem Achilleus (sowie seinem Sohn Neoptolemos) eine tragende Rolle zukommt (Q.S. 14.125142). Dadurch, dass Achilleus und seine Taten nicht nur die narrative Entwicklung entscheidend prägen und bestimmen, sondern auch an zwei herausragenden Eckpunkten der Erzählung - nach seinem Tod und nach der Eroberung Trojas in einem ,Gesang im Gesang', einer mise-en-abyme thematisiert werden, wird dessen prädominante Rolle in den Posthomerica zusätzlich betont.

Zusammenfassend lässt sich also postulieren, dass das Epos des Quintus eigentlich eine Fortsetzung der Ilias mit einem speziellen Fokus auf der Achil-

29 Zur Figur des Neoptolemos und ihren Charakteristika in den Posthomerica vgl. den Beitrag von Bellini Boyten in diesem Band.

30 Geistererscheinungen sind in der klassischen Tragödie keine Seltenheit - man denke etwa an den Geist des toten Dareios in Aischylos' Persai, an Klytaimestra in dessen Eumenides oder an Polydoros in Euripides' Hekabe. In letztgenannter Tragödie wird Achilleus' Geistererscheinung und seine Forderung nach Polyxenas Opferung ebenfalls berichtet, wenn auch nicht inszeniert (vgl. Eur. Hec. 98-153). Wenn also Quintus in seinem Epos den Geist des toten Achilleus auftreten lässt, so hat dies unzweifelhaft eine paratragische Note.

31 Bemerkenswert ist, dass die Tötung von Hektor und von Penthesileia in ein und demselben Vers

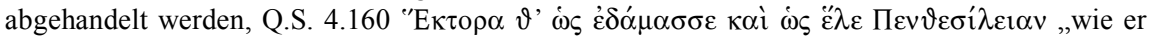
Hektor bezwang und wie er Penthesileia erledigte“. Somit werden die Grenzen zwischen Ilias und Posthomerica verwischt, die Taten des Achilleus, die Achilleia, werden als zusammengehö-

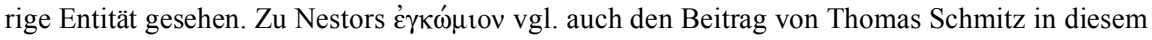
Band. 
leus-Figur und ihrer Taten darstellt, sozusagen eine weitergeführte Achilleia. Zu erwähnen ist hierbei, dass es in der Antike eine ,literaturkritische Strömung' gab, welche sich mit der Frage befasste, weshalb Homer die Ilias nicht analog zur

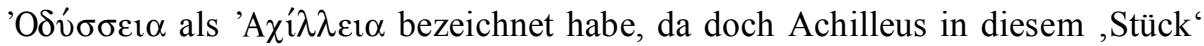
eindeutig die Hauptrolle spiele. ${ }^{32}$ Man könnte somit Quintus' Epos als Ganzes durchaus in einem gewissen Sinne als Stellungnahme zu diesem Diskurs auffassen.

\subsection{Zur Poetologie der einleitenden Verse}

Da ein Proömium das Wesen der Dichtkunst an und für sich thematisiert und reflektiert und da Quintus für sein Epos zwar kein Initialproömium schreibt, jedoch genauso wenig eine simple Fortsetzung der Ilias bietet, sondern vielmehr proömiale Elemente in die ersten 17 Verse seiner ,Einleitung' integriert, wollen wir nun nach expliziten und impliziten poetologischen Aussagen und Anklängen in diesen einleitenden Versen fragen. Drei Aspekte sind m.E. von Bedeutung:

(1) Typologisches Vorbild für die in Kap. 2.1. genannten Szenen im 4. und im 14. Buch der Posthomerica, welche Achilleus' Prädominanz in diesem Epos herausstreichen, ist der Gesang des Demodokos in der Odyssee, der am Hof der Phaiaken den Streit zwischen Odysseus und Achilleus (Od. 8.73-82) sowie die List mit dem hölzernen Pferd und die Zerstörung Trojas (Od. 8.499-520) rezitiert und damit Odysseus, der persönlich zugegen ist, zu Tränen rührt. Eine solche mise-en-abyme reflektiert die Tätigkeit des Homeriden und besitzt somit eine starke poetologische Qualität. Hat man als Leser die poetologische Färbung von Q.S. 4.146-168 und 14.125-142 erkannt und die beiden Szenen mit 1.1-17 gedanklich in Verbindung gebracht, so dürfte dies aus der Retrospektive des lector doctus eine poetologische Lesart auch von 1.1-17 stützen.

(2) Ferner knüpft Quintus in den einleitenden Versen der Posthomerica mehrfach an das Proömium der Ilias an:

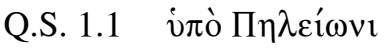
$\sim I l .1 .1 \quad \Pi \eta \lambda \eta \alpha^{\alpha} \delta \varepsilon \omega{ }^{\prime} \mathrm{A} \chi \imath \lambda \hat{\eta} \circ \varsigma$

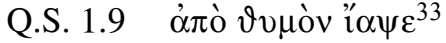

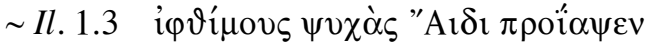
Q.S. $1.14 \tau \grave{\alpha} \pi \rho \hat{\omega} \tau \alpha$
$\sim I l .1 .6 \tau \grave{\alpha} \pi \rho \hat{\omega} \tau \alpha$

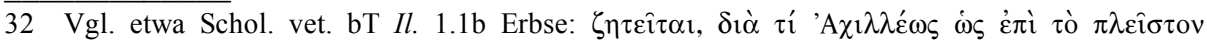

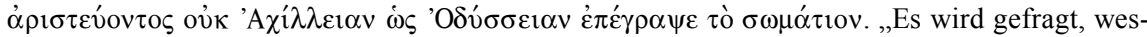
halb er (Homer) seinen Text nicht analog zu ,Odysseia' mit ,Achilleia' überschrieb, da doch Achilleus darin am meisten Heldentaten vollbringt.“

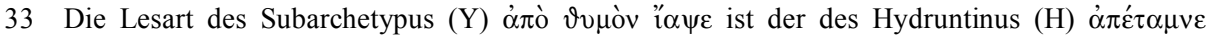

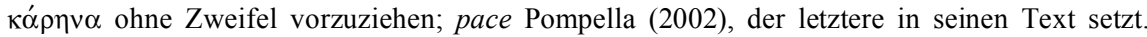

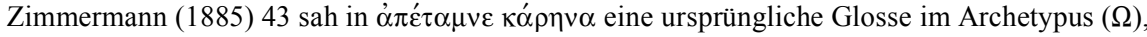
wohl zu Recht. 
Dadurch werden die einleitenden 17 Verse der Posthomerica zusätzlich proömial aufgeladen, ihre oben in Kap. 2.1. diskutierten proömialen Qualitäten werden zusätzlich betont. Dies ist jedoch auch mit weitreichenden poetologischen Implikationen verbunden: ${ }^{34}$ Der Ausdruck $\tau \grave{\alpha} \pi \rho \hat{\omega} \tau \alpha$ verweist nicht nur unüberhörbar auf $I l$. 1.6, sondern er besitzt auch für sich genommen eine implizite poetologische Qualität, insofern als er das Moment des Erinnerns bzw. des Suchens nach den Anfangsgründen - in der Ilias sind dies die Auslöser für den Streit zwischen Agamemnon und Achilleus, in den Posthomerica die Anfänge von Achilleus' Wüten und Morden - als Movens epischen Erzählens in den Vordergrund stellt. Diese Suche nach den Anfangsgründen ist insbesondere auch in den homerischen Götterhymnen sehr präsent: eine Mehrheit der Hymnen beginnt mit einer Form des Verbs $\alpha \rho \chi \varepsilon \sigma \vartheta \alpha \iota$,,beginnen“ oder einer Futurform von $\alpha \varepsilon i ́ \delta \varepsilon ı$,,besingen“. 35 Vergleichbares gilt auch und ganz besonders für den Ausdruck $\mu \nu \eta \sigma \alpha ́ \mu \varepsilon v o r$ $\pi \rho о \tau \varepsilon ́ \rho \omega v$ in Q.S. 1.9, insofern als das Erinnern, das Nicht-dem-VergessenAnheimstellen ja die eigentliche Hauptfunktion des epischen Erzählers bzw. der ihn unterstützenden Musen ist. Die Musen gelten bei und seit Hesiod als Töchter der personifizierten Mnemosyne ${ }^{36}$ und sind mit hoher Wahrscheinlichkeit auch etymologisch mit der indogermanischen Wurzel * mneh $_{2^{-}}$,,denken an“ in Verbindung zu bringen. ${ }^{37}$ Das Verb $\mu \nu \eta \sigma \hat{\alpha} \sigma \vartheta \alpha \iota$ und seine Verwandten sind also mit

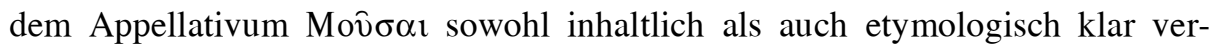

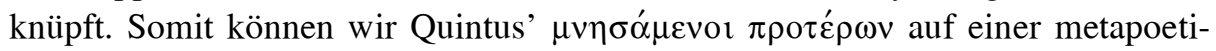
schen Ebene als (strukturelles und inhaltliches) Substitut für die zu erwartenden, jedoch nicht vorhandenen Musen zu Beginn der Posthomerica auffassen. ${ }^{38}$

34 Herrn Prof. Dr. Christoph Riedweg sei für den wertvollen Hinweis auf diesen Aspekt herzlich gedankt.

35 Am gebräuchlichsten ist die Versschlussformel ’’ $\rho \chi о \mu$ ' $\alpha \varepsilon i \delta \varepsilon ı v$,ich fange an zu besingen“

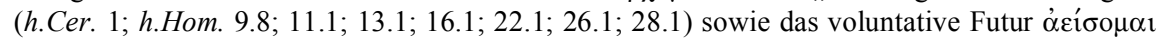
„ich will besingen“" (h.Hom. 10.1; 15.1; 23.1; 30.1; ausserdem h.Ven. 2 ö $1 \sigma o \mu \alpha$ ).

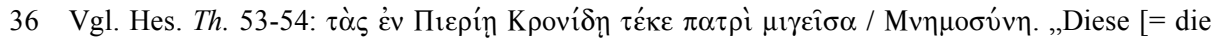
Musen] gebar Mnemosyne in Pierien, nachdem sie mit ihrem Vater, dem Kronos-Sohn, geschlechtlich verkehrt hatte."

37 Zur Wurzel und ihren verbalen Ableitungen im Griechischen vgl. LIV s.v. ${ }^{*} m n e h_{2^{-}}$. Zur Etymologie von Mov̂ $\alpha \alpha<{ }^{*} m o n t^{h} i a$ vgl. Frisk II s.v. $\mu$ ov̂ $\sigma \alpha$; LfgrE s.v. Mov̂ $\sigma \alpha$. Diese Herleitung ist wahrscheinlicher als die von Jakob Wackernagel angenommene Etymologie Mov̂ $\sigma \alpha$ $<$ *montia „Bergnymphe" (zu lat. mons), da dies im Griechischen ein völlig isoliertes Wort wäre (vgl. Frisk a.a.O.).

38 Vgl. auch den Eingangsvers zum homerischen Apollonhymnos, h.Ap. 1: $\mu v \eta \dot{\sigma o \mu \alpha \iota ~ o v ̉ \delta \varepsilon ́ ~}$

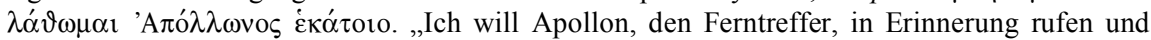
nicht dem Vergessen anheimstellen.“ - Eine Kombination des für die Anfänge homerischer Göt-

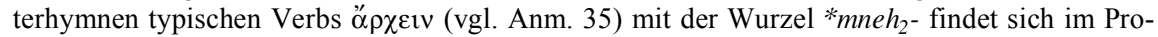

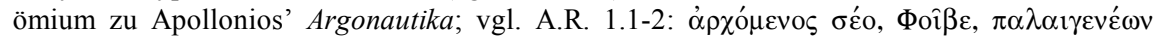

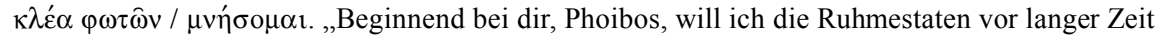
geborener Männer in Erinnerung rufen.“ 
(3) Schliesslich ist auch der Verzicht auf ein Initialproömium mit weitreichenden poetologischen Implikationen verbunden: Einerseits schliesst Quintus zwar motivisch und narrativ an die Ilias an, andererseits und untrennbar damit verbunden setzt er sich jedoch genau durch diesen Verzicht ebenso klar von der Gattungstradition, die einen Musenanruf für ein Heldenepos zwingend verlangt, ab. Der Kunstgriff besteht also darin, mittels einer formalen Leerstelle einen direkten Anschluss an das Vorbild und eine Absetzung davon aneinanderzukoppeln. Unser Dichter bedient sich also nicht der Technik reiner imitatio, sondern der aemulatio bzw. einer imitatio cum variatione. Dies lässt sich bis in sprachliche Details hinein beobachten: Der 1. Vers der Posthomerica knüpft nicht nur inhaltlich an das Ende der Ilias an, sondern er umklammert auch sprachlich deren

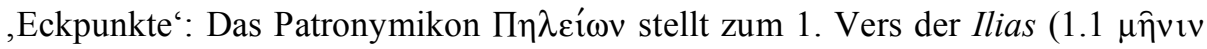

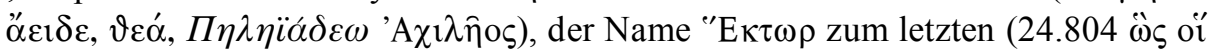

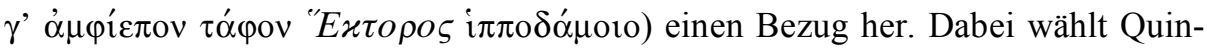
tus jedoch ein anderes Suffix zur Bildung des Patronymikons für Achilleus

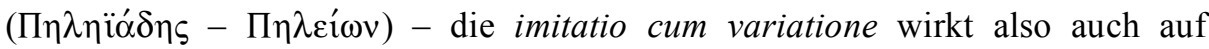
sprachlicher Ebene: Einerseits wird die Ilias als Ganzes in den Blick genommen, andererseits findet gleichzeitig eine Abgrenzung von ihr statt. Möglicherweise

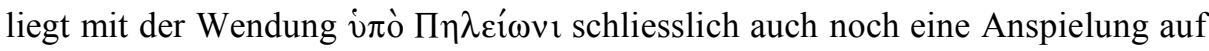
einen alternativen Anfang der Ilias vor, welcher gemäss Aristoxenos im Schwange war und drei Verse anstelle von Il. 1.1-9 umfasste, denn dort kommt dasselbe Patronymikon ebenfalls vor. ${ }^{39}$ Falls eine solche Anspielung vorliegen sollte, so liesse sich für Quintus erneut (s.o. Kap. 2.1. a.E.) eine gewisse Auseinandersetzung mit der Homerexegese und -philologie postulieren, was im Kontext der Zweiten Sophistik nicht untypisch wäre. ${ }^{40}$

Der Verzicht auf ein Initialproömium ermöglicht also, zusammenfassend gesagt, nicht bloss eine optimale, quasi-dramatische Vorbereitung und Inszenierung des nun folgenden Geschehens (s.o. Kap. 2.1.), sondern ihm kommt auch eine

39 Aristox. fr. 91, I Wehrli; vgl. dazu zusammenfassend Kirk (1985) 52-53. Die drei Verse lauten:

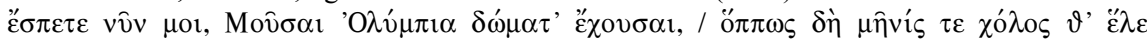

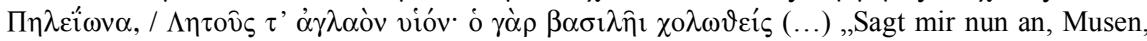
die ihr die Häuser des Olymps bewohnt, wie Groll und Zorn den Peleus-Sohn ergriffen und Letos strahlenden Sohn: denn der, dem Führer zürnend [...]“ - Zum 1. Vers ह̌ $\sigma \pi \varepsilon \tau \varepsilon \kappa \tau \lambda$. vgl. auch Kap. 3.1.

$40 \mathrm{Zu}$ denken ist hierbei an die Ablehnung der (über-)kritischen Homerphilologie in der Zweiten Sophistik. Von Triphiodor ist bekannt, dass er als , anti-alexandrinischer' poeta doctus dichtet, indem er Lesarten, welche die Alexandriner ablehnten, in seinen Text aufnimmt; vgl. Dubielzig (1996) 16-18. Lukian macht sich in den Verae Historiae über Zenodot und Aristarch lustig, indem er Homer persönlich auf der Insel der Seligen die Echtheit sämtlicher ihm zugeschriebenen

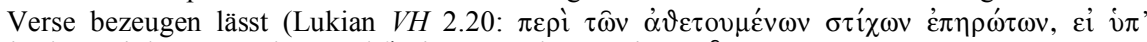

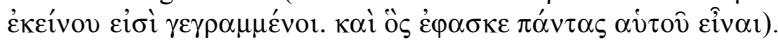


implizit poetologische Aussage zu, dergemäss sich Quintus mittels einer formalen Leerstelle als Homerus novus in Szene setzt. Der Schlüssel zu einer adäquaten Erklärung des unkonventionellen Beginns der Posthomerica liegt somit nicht in einem mono-, sondern in einem bikausalen Deutungsansatz.

\section{Das Binnenproömium, seine Intertextualität und poetologischen Implikationen}

Das 12. Buch der Posthomerica hat die berühmte Geschichte vom hölzernen Pferd zum Thema: von der göttlich inspirierten Idee des Odysseus über den Bau durch Epeios bis zur Einholung durch die Trojaner; dazwischen die bekannten Episoden mit dem freiwillig zurückgebliebenen Sinon und dem von Athene hart bestraften und geschundenen Laokoon. Vor dem Katalog der Helden, welche das Ross besteigen (12.314-335), schaltet Quintus einen Musenanruf mit folgendem Wortlaut ein:

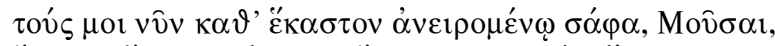

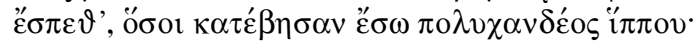

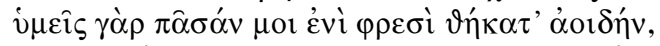

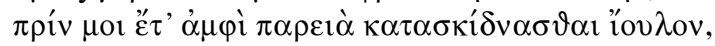

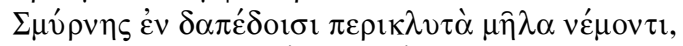

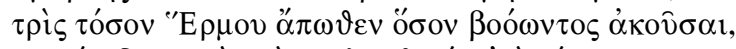

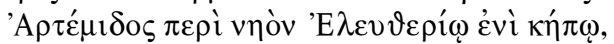

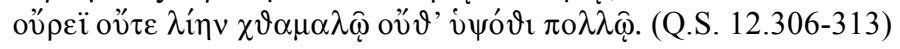

Diese nun nennt mir, der ich «euch danach〉 frage, Musen, untrüglich der Reihe nach, welche in das vielfassende Pferd hineinstiegen:

Denn $i h r$ habt mir die ganze Sangeskunst in den Sinn gelegt, noch bevor mir meine Wange ringsum den Bartflaum wachsen liess, als ich in Smyrnas Landen das vielberühmte Vieh weidete, 〈nur〉 dreimal so weit vom Hermos entfernt, wie 〈man〉 einen Rufenden hören «kann〉, in der Umgebung eines Artemistempels im Garten der Freiheit 〈an einem Ort〉 weder von allzu flacher Anhöhe noch besonders steil.

Im Wesentlichen sind es drei Subtexte - ein homerischer, ein hesiodeischer und ein kallimacheischer -, welche den Musenanruf des Quintus prägen und deren Funktionen im Folgenden untersucht werden sollen. Dabei ist vorauszuschicken, dass die fraglichen, Vorbildtexte' in der Forschung seit langem bemerkt und aufgelistet wurden; ${ }^{41}$ was m.E. jedoch bis anhin fehlt, sind Überlegungen, wie die

41 Vgl. Vian (1969) 101 Anm. 1; Campbell (1981) 101-105; Gärtner (2005) 23 Anm. 5. 
vorliegenden intertextuellen Beziehungen für das Verständnis von Quintus' Musenanruf interpretatorisch fruchtbar zu machen sind.

\subsection{Homer}

Erster intertextueller Bezugspunkt ist das Binnenproömium im 2. Buch der Ilias vor dem berühmten Katalog der Schiffe:

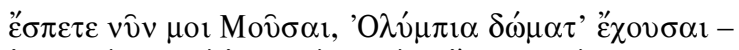

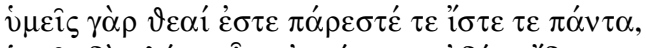

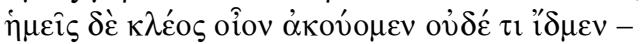

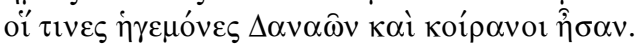

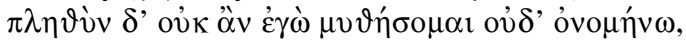

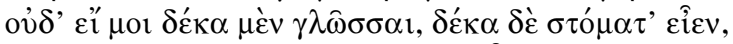

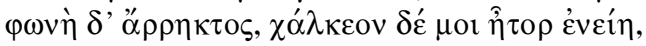

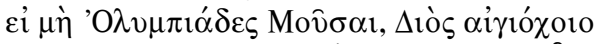

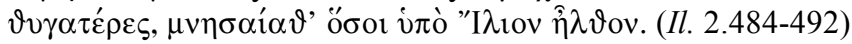

Sagt mir nun an, Musen, die ihr die Häuser des Olymps bewohnt

- denn ihr seid Göttinnen und seid zugegen und wisst alles,

wir aber hören von der Kunde nur, wissen sie aber nicht «mit Sicherheit - ,

welches die Anführer und Herren der Danaer waren:

Denn die 〈ganze〉 Menge könnte ich wohl nicht erzählen und nennen,

auch wenn ich zehn Zungen, zehn Münder hätte

und eine unzerreissbare Stimme und 〈auch wenn〉 mir ein ehernes

Herz innewohnte,

wenn nicht die Musen vom Olymp, die Töchter des Aigishalters Zeus,

mir in Erinnerung riefen, welche 〈Helden` nach Ilion zogen.

Quintus greift den homerischen Musenanruf in mehrfacher Weise auf:

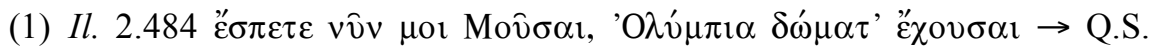

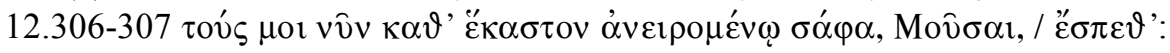

Es handelt sich bei Il. 2.484 um einen Iteratvers, der in der Ilias drei weitere Male begegnet. ${ }^{42}$ An diesen Stellen jedoch finden sich keine ausgedehnten Binnenproömien, sondern nur kurze ,Stossgebete', die sich aus der Formelhaftigkeit der oral poetry erklären lassen. Die enge Verknüpfung von Q.S. 12.306-313 mit Il. 2.484-492 wird deswegen also in keiner Weise beeinträchtigt. Allerdings darf der iliadische Vers wohl als ,Prototyp“ oder,Vorzeigemodell' eines epischen Musenanrufs gelten, der ausserdem in Hesiods Theogonie in leicht variierter

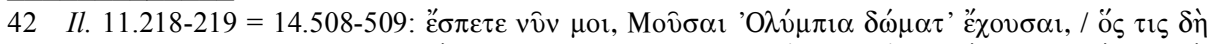

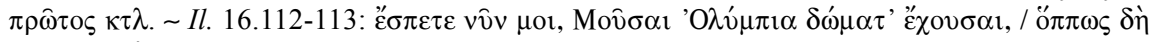
$\pi \rho \hat{\tau} \tau \mathrm{T} \kappa \tau \lambda$. 
Form vorliegt ${ }^{43}$ und auch das durch Aristoxenos überlieferte, aus drei statt neun Versen bestehende ,alternative' Ilias-Proömium einleitet (s.o. Anm. 39). Im Kern beibehalten ist hier die homerische Formel "ै $\sigma \pi \varepsilon \tau \varepsilon v \hat{v} \nu \mu o \imath$ Mov̂ $\sigma \alpha$, sie wird jedoch von Quintus in zweifacher axialsymmetrischer Anordnung gespiegelt und somit variiert: "

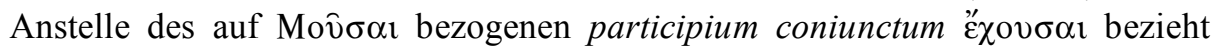

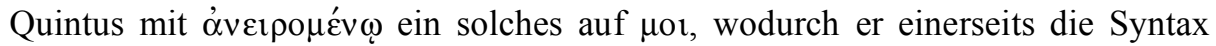
gegenüber der Vorlage variiert und andererseits - rein sprachlich - eine gewisse Gewichtsverschiebung von den Musen hin zum epischen ,Ich'vornimmt. Dabei

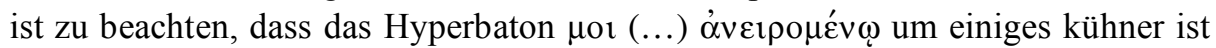

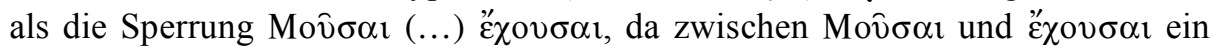
zum participium coniunctum gehöriges Satzglied steht, was bei $\mu$ or (...)

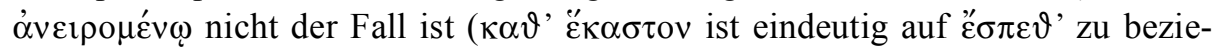
hen). ${ }^{44}$ Vollständig neu und somit auffällig sind sodann die quantitative Angabe

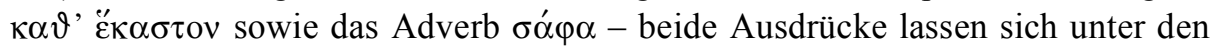
Oberbegriff ,Genauigkeit' subsumieren und haben also die Funktion, die Musen nicht bloss um eine approximative, sondern um eine akkurate Eingebung zu bitten. $^{45}$ Ebenfalls neu hinzugekommen ist das Determinativpronomen $\tau$ oú $\zeta$, welches im darauf folgenden Vers durch den Relativsatz ö $\sigma o r \kappa \tau \lambda$. expliziert wird. Dieser Zusatz erscheint mir weder zufällig noch unerheblich, zumal die syntaktisch auffällige Anfangsstellung an sich schon eine besondere Bedeutung impliziert: Die Initialposition des Akkusativobjekts als des zu besingenden Gegenstands ist so charakteristisch für die beiden homerischen Epen (Il. 1.1

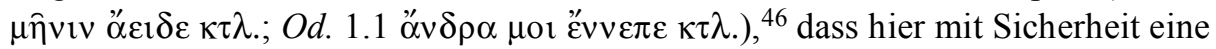
konkrete Bezugnahme darauf vorliegt.

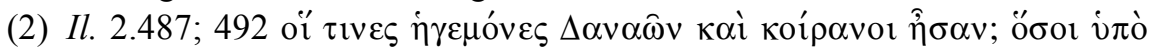

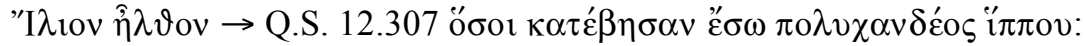

Überträgt man die strukturelle Analogie von Il. 4.487 bzw. 492 und Q.S. 12.307 auf den Inhalt, so drängt sich die Schlussfolgerung auf, dass nicht irgendwelche Männer, sondern nur die besten das hölzerne Pferd besteigen, was sich im

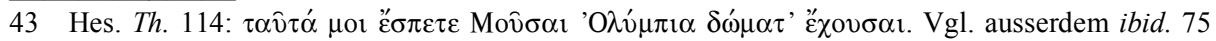

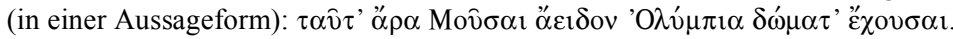

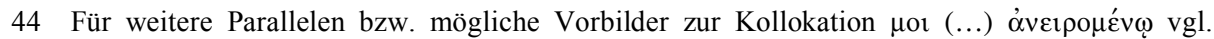
Campbell (1981) 103.

45 Zur Funktion der beiden Ausdrücke im Zusammenhang mit den Musen als ,potentiellen Lügnerinnen's.u. Kap. 3.3., mit Anm. 72.

46 Vgl. Klotz (1965) 14: „Der grammatische Kasus macht den Zorn und den Mann zum greifbaren Objekt der Erzählinstanz, zum betroffenen Gegenstand der Erzählung. Sie werden buchstäblich ,akkusativ ‘ vors Tribunal der epischen Veranstaltung zitiert, zunächst nur lapidar und namentlich - dann erst, im Lauf des erzählerischen Prozesses, wird die Geschichte in aller sinnlichen Ausführlichkeit entwickelt.“ 
darauf folgenden Katalog dann ja bestätigt. ${ }^{47}$ Quintus' hauptsächliche Variation besteht darin, die beiden homerischen Teilsätze auf nur einen Relativsatz zu verkürzen. Diese Verkürzung steht in einem gewissen Gegensatz zu der Erweiterung, welche der iliadische Vers 2.484 in seiner variatio bei Quintus erfährt. Die beiden Teile (Musenanruf - Benennung des zu besingenden Gegenstands) verhalten sich also quantitativ umgekehrt proportional zueinander.

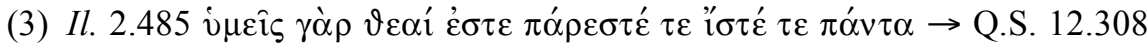

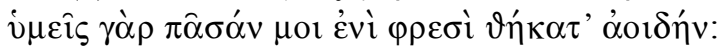

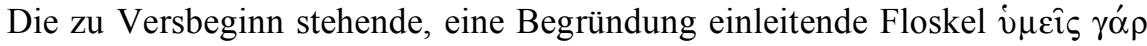
hat Quintus übernommen, um sie sogleich inhaltlich zu variieren: In der Ilias geht es darum, dass die Musen im Gegensatz zu den Menschen bzw. den menschlichen Dichtern alles wissen und alles zu künden vermögen, weshalb der Sänger ihrer Hilfe bedarf. Quintus dagegen verlagert den Fokus weg von den Musen hin zum epischen ,Ich`: Nicht die Autorität der Musen wird von ihm thematisiert, sondern das Können des Sängers - welches jener freilich den Musen verdankt. Somit findet auf ebenso unerwartete wie auffällige Weise der Übergang vom homerisch inspirierten Musenanruf, der sich im Wesentlichen auf die Bitte des Dichters um göttliche Inspiration bzw. Unterstützung beschränkt, zum hesiodeisch geprägten Topos der Musenweihe des Dichters statt (s.u. Kap. 3.2.). ${ }^{48}$ Der Übergang ist insofern besonders kunstvoll gestaltet, als da er rein gedanklich völlig glatt ver-

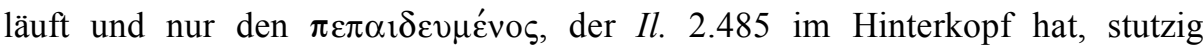
macht. ${ }^{49}$ Doch mehr noch als dies: Quintus' Satzstruktur ist in der Tat konziser als die homerische, handelt es sich doch bei $\mathrm{Il}$. 2.485-486 um eine zwischen die Verse 484 (Bitte um Inspiration bzw. Unterstützung) und 487 (Benennung des zu besingenden Gegenstands) eingeschobene Parenthese, welche Quintus durch die

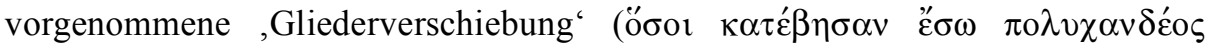

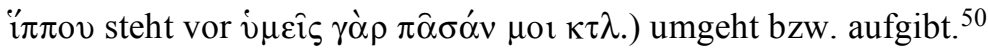

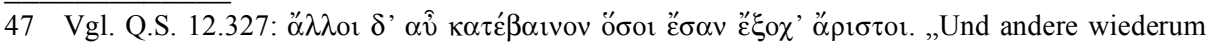
stiegen ein, welche die allerbesten waren." Vgl. auch Anm. 52.

48 Dass der Gedankengang in eine andere Richtung geht, wird spätestens in Q.S. 12.309 deutlich, denn dort fehlt das Pendant zum homerischen $\dot{\eta} \mu \varepsilon \hat{\imath} \varsigma \delta \grave{\varepsilon} \kappa \tau \lambda$. (Il. 2.486), welches in Antithese zu i $\mu \varepsilon i \varsigma \zeta \gamma \grave{\alpha} \rho \kappa \tau \lambda$. steht.

49 Der Homerkenner, der Il. 2.485 als Subtext mitliest, wird irregeführt, während ein unkundiger Leser die Irreführung gar nicht bemerkt bzw. nicht als solche wahrnimmt. Dies spricht m.E. dafür, dass Quintus' Musenanruf auf zwei Ebenen gelesen werden darf: auf einer ,naiven“ einerseits, auf einer ,gebildeten' andererseits. Somit scheint bereits ein Hinweis darauf gegeben, dass die in Kap. 4. zu postulierende poetologische Lesart des ganzen Musenanrufs zulässig ist, da die Leserlenkung von Beginn weg in diese Richtung geht.

50 Weitere, kleinere imitatorische Bezüge zu Il. 2.485 nimmt Quintus durch die Form $\pi \hat{\alpha} \sigma \alpha v$

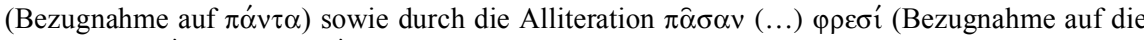
Alliteration $\left.\pi \alpha ́ \alpha \varepsilon \sigma \tau \varepsilon[\ldots] \pi \alpha^{\prime} \nu \tau \alpha\right)$ vor. 


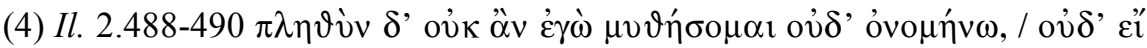

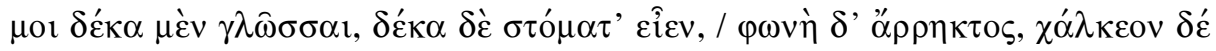

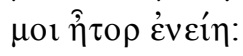

Diese Verse des iliadischen Vorbilds haben keine konkrete Entsprechung bei Quintus. Doch auch diesem Umstand ist Beachtung zu schenken, da ja, wie wir im Zusammenhang mit dem nicht-vorhandenen Initialproömium gesehen haben, auch ein ,Null-Signal' semiotisch bedeutsam sein kann. Intertextuelles Lesen funktioniert nicht in erster Linie über das Wiedererkennen einzelner Wörter, Floskeln oder Sätze, sondern vor allem und wesentlich über die mentalassoziative Verknüpfung zusammenhängender Stellen und gedanklicher Komplexe. Wer beim Lesen des Musenanrufs in den Posthomerica an das genannte Binnenproömium der Ilias denkt, dürfte sich die ganze Stelle in Erinnerung rufen - und wird die formale Leerstelle, welche die fehlende, Verarbeitung ' von Il. 2.488-490 in den Posthomerica hinterlässt, bemerken. Meines Erachtens lässt sich diese Leerstelle folgendermassen deuten: Der Leser soll selber die strukturellnarratologische Analogie zwischen den beiden Passagen bemerken, welche darin besteht, dass in beiden Fällen ein Musenanruf vor einem ebenso bedeutsamen wie schwierig zu memorierenden Katalog im Sinne eines ,Stossgebets' den Dichter unterstützen soll: dort die griechischen Kämpfer vor ihrer Abfahrt nach Troja, hier die Helden vor ihrem Einstieg ins hölzerne Pferd. ${ }^{51}$ Damit ist unweigerlich eine weitere zentrale Frage angetönt, nämlich, weshalb Quintus ausgerechnet hier den ,obligaten' Musenanruf ,nachliefert'. Die Antwort ist: weil die Besteigung des Pferdes einen ähnlich wichtigen Moment darstellt wie die Abfahrt der Griechen. ${ }^{52}$ Oder, narratologisch gesprochen: Weil es sich in beiden Fällen um eine strukturell analoge Stelle handelt, deren richtiges Memorieren zentral ist und hier treffen sich Narratologie und Mythos - den Ausgang des Geschehens unter Umständen wesentlich beeinflusst. ${ }^{53}$ Poetologisch gelesen, besagt die Leerstelle somit kaum etwas anderes, als dass unser Dichter nicht bloss weiss, wie man einen veritablen Musenanruf zu verfassen hat, sondern auch, wo er hingehört. In einem weiteren, eher assoziativen als streng logischen Sinne haben Il. 2.488-490 freilich doch eine gewisse Entsprechung bei Quintus: Einerseits ist da das Adjek-

51 Hinzu kommt, dass der Katalog der Helden, welche das Pferd besteigen, natürlich von ungleich geringerer Quantität ist als der iliadische Schiffskatalog. Es käme somit einer Selbstironisierung gleich, würde Quintus hier eine Aussage im Stile von Il. 2.489-490 einfügen.

$52 \mathrm{Zu}$ erwähnen ist hierbei, dass Quintus nebst den ungenannten Griechen (Q.S. $12.327 \alpha^{\prime} \lambda \lambda \lambda_{0} \delta$ '

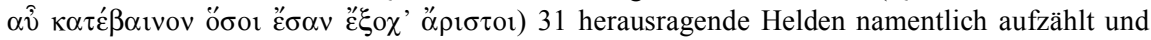
somit den längsten aller aus der Antike tradierten diesbezüglichen Kataloge liefert; vgl. dazu Campbell (1981) 101-102; Gärtner (2005) 174. Ausserdem fällt auf und ist bestimmt kein Zufall, dass 23 der 31 Helden in Quintus' Katalog aus dem homerischen Schiffskatalog, entlehnt` sind; für Details hiezu vgl. Keydell (1963) 1287.

53 Paradox formuliert: Wenn der Dichter nicht alle oder die falschen Helden nach Troja absegeln oder ins Pferd einsteigen lässt, besteht die Gefahr, dass die Griechen scheitern. 


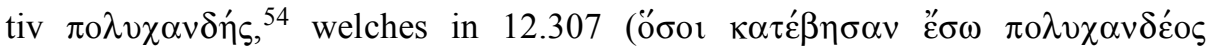
"i $\pi \mathrm{ov})$ das Moment der ,riesigen Menge‘ in einem Wort zum Ausdruck bringt und somit einen gewissen Bezug zu den drei Ilias-Versen herstellt. Andererseits hat die ausführliche topographische Beschreibung in Q.S. 12.310-313 in Il. 2.488490 im allerweitesten Sinne ein Vorbild, als da an beiden Stellen eine auffällige Digression innerhalb eines Musenanrufs vorliegt.

Zusammenfassend lässt sich festhalten, dass Quintus sein homerisches Vorbild Il. 2.484-492 im Sinne einer imitatio cum variatione sprachlich-stilistisch aufgreift und variiert, im Sinne einer aemulatio punkto ,Glätte ‘ ,verbessert' (!) sowie - damit verknüpft bzw. daraus resultierend - dem bei Homer noch gänzlich fehlenden Thema der dichterischen Musenweihe zugänglich macht, womit er den intertextuellen Zugang zu Hesiod eröffnet und eine Gewichtsverschiebung weg von den Musen hin zur Rolle des epischen ,Ichs“ vornimmt oder zumindest impliziert, wodurch einer poetologischen Lesart von Q.S. 12.306-313 der Weg geeebnet wird. Gleichzeitig macht der intertextuelle Bezug zu der genannten Ilias-Stelle als Ganzes deutlich, dass Quintus sein Binnenproömium am wohl passendsten Ort in seinem Epos angebracht hat, was Zeugnis für seine Fähigkeiten als homerisierender Dichter ablegt. ${ }^{55}$

\subsection{Hesiod}

Als zweiter intertextueller Bezugspunkt ist die sog. ,Musenweihe' in Hesiods Theogonie zu nennen: Ein ,Ich'-Sprecher, der sich Hesiod nennt, erzählt, die Musen seien ihm erschienen und hätten ihm die Fähigkeit zum Singen eingegeben, als er am Fusse des Berges Helikon die Schafe weidete:

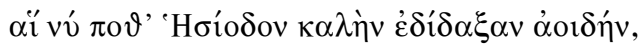

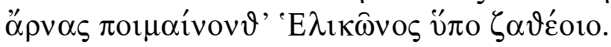

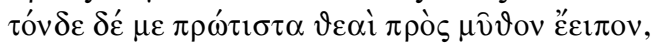

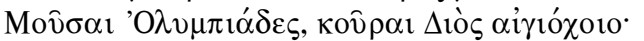

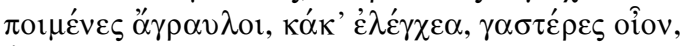

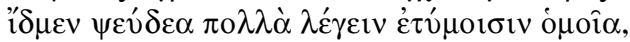

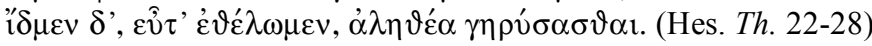

Diese (= die Musen) lehrten einst Hesiod die schöne Sangeskunst, als er die Schafe weidete am Fusse des hochhehren Helikon.

Und dieses Wort sprachen die Göttinnen bei der ersten Begegnung zu mir,

die Musen vom Olymp, die Töchter des Aigishalters Zeus:

54 Zur Polyvalenz des Adjektivs $\pi 0 \lambda \nu \chi \alpha \nu \delta \eta ́ \varsigma$ an dieser Stelle s.u. Kap. 4.2.

55 Davon, dass ,das Empfinden für die verschiedene〈n〉 Arten der Dichtung, die von Homer und Hesiod vertreten werden“, „durch diese Vermischung erloschen“ sei, wie Koster (1970) 156 behauptet, kann also nicht die Rede sein. 
„Ihr Hirten, die ihr das freie Feld bewohnt, ihr üblen Feiglinge, nur Bäuche!

Wir wissen viele Trugreden zu sagen, der Wahrheit ähnlich,

wir wissen aber auch, wenn wir es wollen, Wahres zu verkünden." ${ }^{56}$

Die Passage, die Hesiods Musenweihe am Helikon schildert, ist von Quintus in den Versen 308-310 verarbeitet worden. Wörtlich übernommen ist allerdings nur

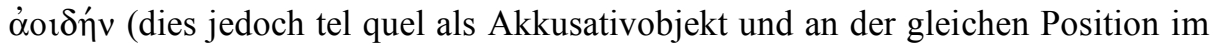
Vers) ${ }^{57}$ das Prädikativum $\pi \hat{\alpha} \sigma \alpha v$ kommt dabei einer aemulatio gegenüber dem Attribut $\kappa \alpha \lambda \eta \dot{v} v$ gleich (Hesiods Gesang ist ,nur“ „,schön“, der des Quintus da-

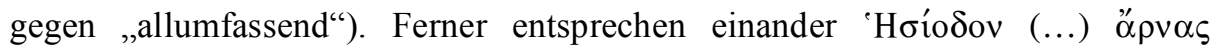

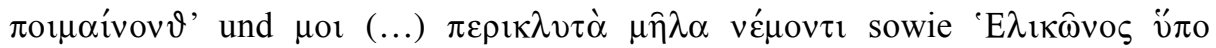

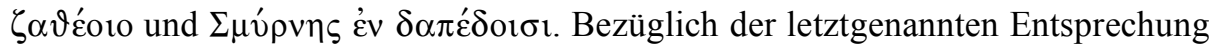
ist bemerkenswert, dass die Musenweihe bei Hesiod in der Abgeschiedenheit an einem Berg, diejenige bei Quintus aber in der Nähe einer Stadt im Flachland stattfindet. Die Gegensätze (Land-Stadt; Einsamkeit-Betriebsamkeit; BergFlachland) könnten eigentlich nicht grösser sein. Somit fällt Quintus' topographi-

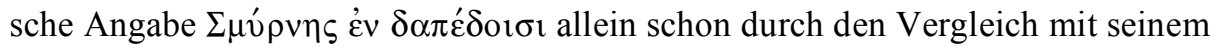
Vorbild aus dem Rahmen. Die Heiligkeit des Ortes sodann, die im Adjektiv $\zeta \alpha \vartheta$ ćoto enthalten ist, ist bei Quintus ausgelagert auf eine andere topographische

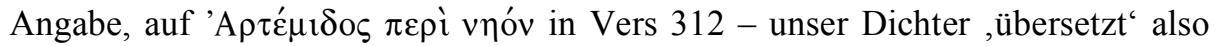
sein Vorbild nicht tel quel phrasenweise, sondern er fächert einen einzelnen

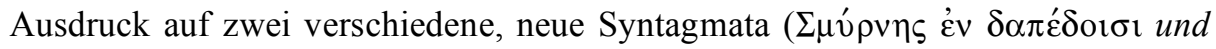
'A $\tau \tau \dot{\mu} \mu 1 \delta$ о $\varsigma \varepsilon \rho i$ vๆóv) auf. Vers 309 schliesslich ist ohne hesiodeische Vorlage, bedeutet also eine Erweiterung oder, wenn man so will, eine aemulatio gegenüber dieser und beruht inhaltlich auf einem kallimacheischen Topos (s.u. Kap. 3.3.). Quelle ist $O d .11 .319$ gemäss Campbell (1981) 104; allerdings beschränkt sich die Intertextualität in diesem Fall auf eine sprachliche Formulierung, die in beiden Fällen einer Altersangabe dient. ${ }^{58} \mathrm{Da}$ jedoch diese Odyssee-Stelle nach Campbell a.a.O. „rise to a host of imitations“ gab (Stellen vgl. ibid.), ist es durchaus mög-

56 Zur Musenweihe am Helikon (Hes. Th. 22-34) vgl. West (1966) 158-167 und Verdenius (1972) 233-240.

57 Dadurch, dass es sich um die einzige tel-quel-Adaption handelt, wird die Schlüsselfunktion des Wortes óor $\delta$ ' herausgestrichen. Zur vorliegenden Bedeutung „Sangeskunst“ (hier wohl eher als „Gesang“) vgl. Verdenius (1972) 233; Campbell (1981) 103.

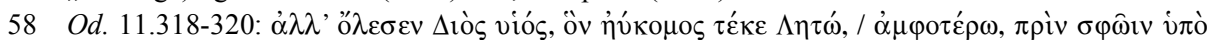

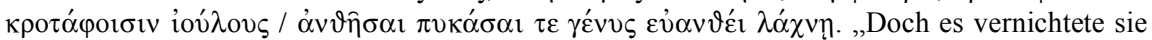
der Sohn des Zeus, den die schönhaarige Leto geboren hatte, und zwar〉 beide, bevor ihnen unter den Schläfen der Bartflaum blühte und die Kinnbacken mit schönspriessendem Haar bedeckte.“ - Der Kontext ist freilich ein völlig anderer: Es geht hier um Otos und Ephialtes (Söhne des Poseidon und der Iphimedeia), welche von Apollon in der Blüte ihrer Jugend getötet werden, weil sie den Göttern gedroht haben, den Olymp zu ersteigen. 
lich und plausibel, dass Quintus die Passage ebenfalls memoriert und somit die imitatio Hesiodi mit einem homerischen Element angereichert hat.

Hinzuweisen ist in diesem Zusammenhang auf eine weitere Stelle in den Posthomerica, an welcher die Musen auftreten: Nach Achilleus' Tod im 3. Buch kommen diese vom Helikon, um Thetis bei der Trauer um ihren gefallenen Sohn beizustehen. ${ }^{59}$ Quintus lokalisiert also die Musen durchaus in hesiodeischer Tradition auf ihrem ,Hausberg “ - umso klarer tritt zutage, dass es mit der

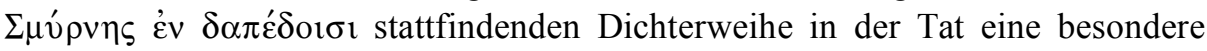
Bewandtnis hat. Sodann richtet sich die Muse Kalliope in einer längeren consolatio an Thetis (Q.S. 3.633-654). Zweierlei scheint mir dabei für unsere Zwecke von Belang: Einerseits ist der Umstand, dass „Kalliope persönlich“ spricht, ${ }^{60}$ zweifellos eine weitere Referenz auf Hesiods Theogonie, denn dort gilt selbige als „,vortrefflichste aller 〈Musen〉“. ${ }^{61}$ Andererseits besitzt Kalliopes consolatio ebenfalls poetologische Qualität, denn in Q.S. 3.645-647 tröstet sie Thetis damit, dass Achilleus in Zukunft dank ihrer und der anderen Musen Inspiration von den Dichter-Sängern ( $\alpha$ oı $\delta$ oí) auf Erden besungen werden wird ${ }^{62}$ - genau dies aber ist ja in dem Moment, in dem man die nämlichen Verse liest oder hört, der Fall, es handelt sich also um eine mise-en-abyme. Somit sind die Musen und Hesiods Theogonie an einer anderen, früheren Stelle der Posthomerica mit Quintus' Poetologie ebenfalls fest verknüpft.

\subsection{Kallimachos}

Als dritter und letzter intertextueller Bezugspunkt erscheint der alexandrinische poeta doctus Kallimachos von Kyrene und sein berühmter, wenn auch nur fragmentarisch erhaltener Aitienprolog, welcher, soweit dies ersichtlich ist, aus dem sog. ,Telchinengedicht' ${ }^{6}$ und einer Traumszene besteht. ${ }^{63}$ In Letzterer berichtet

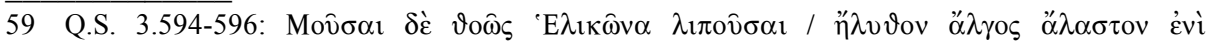

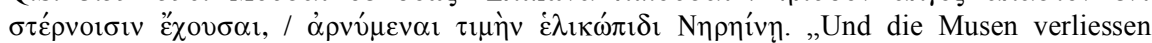
geschwind den Helikon, unvergesslichen Schmerz in ihren Herzen tragend, und kamen, der glanzäugigen Nereus-Tochter (= Thetis) die Ehre zu erweisen.“ - Zur Topik der ganzen Szene vgl. Vian (1963) 172 Anm. 4; James (2004) 286.

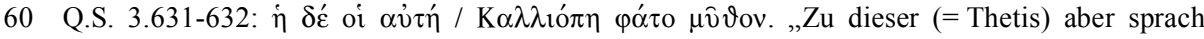
Kalliope persönlich ein Wort.“

61 Hes. Th. 79: $\ddot{\eta} \delta \grave{\varepsilon} \pi \rho \circ \varphi \varepsilon \rho \varepsilon \sigma \tau \alpha \dot{\tau} \tau \eta \dot{\varepsilon} \sigma \tau \grave{\nu} \dot{\alpha} \pi \alpha \sigma \varepsilon \dot{\varepsilon} \omega v$. „Diese aber (= Kalliope) ist die vortrefflichste aller «Musen〉."

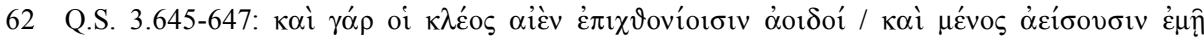

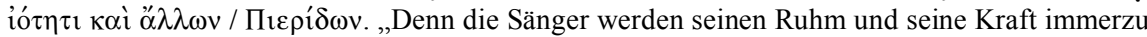
für die 〈Menschen〉 auf Erden besingen durch meinen und der anderen Pieriden Willen.“

63 Telchinengedicht: Call. Aet. 1 fr. 1.1-40 Pfeiffer (= 1 Asper); Traumszene: Call. Aet. 1 fr. 1.4145 und 2 Pfeiffer ( $=3$ und 4 Asper). 
Kallimachos, dass ihm träumte, er werde von Kyrene aus auf den Helikon versetzt und empfange dort wie einstmals Hesiod die Musenweihe. ${ }^{64} \mathrm{Da}$ es sich um Fragmente handelt, dürfen Vergleiche mit Quintus' Musenanruf und erst recht Schlussfolgerungen daraus nur mit höchster Vorsicht vorgenommen werden. Gleichwohl lassen sich m.E. einige Berührungspunkte anführen:

(1) Kallimachos soll seine Musenweihe als Jüngling empfangen haben. Dies geht zwar aus den erhaltenen kallimacheischen Fragmenten nur indirekt hervor, doch dürfte die spätere diesbezügliche Überlieferung keine Neuerfindung sein. ${ }^{65}$ Ausserdem passt dies zu Kallimachos' Anspruch, mit seiner Dichtung etwas grundlegend Neues zu schaffen. ${ }^{66}$ Vers 309 von Quintus' Musenanruf, als dessen sprachliche Vorlage sich ein Odyssee-Vers ausmachen liess (s.o. Kap. 3.2.), geht also inhaltlich auf einen kallimacheischen Topos - den Topos der Dichterweihe im Jugendalter - zurück und assoziiert somit gleichzeitig die Vorstellung von einem ,frischen und unverbrauchten Dichterling', der die ausgetretenen Pfade verlässt und etwas Neues wagt.

(2) Kallimachos nimmt mit seiner Musenweihe eindeutig auf ebenjene des Hesiod und somit auf den entsprechenden hesiodeischen Subtext (Th. 22-28) Bezug. ${ }^{67}$ Die Art und Weise von Kallimachos' Auseinandersetzung mit seinem Vorbild kann und soll hier nicht erörtert werden ${ }^{68}$ - für unsere Zwecke mag der Umstand genügen, dass Kallimachos sich in kreativer Weise mit seinem epischen

64 Ein wichtiges Zeugnis, welches den Traum und die Dichterweihe des Kallimachos als Voraussetzung und Beginn seiner Aitien erwähnt, ist $A P$ 7.42: Dort wird der ,grosse und weitberühmte

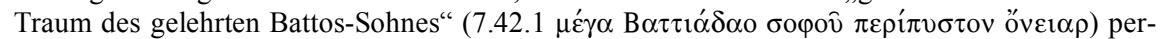
sonifiziert angesprochen. - Für eine Zusammenstellung aller griechischen und lateinischen Zeugnisse zu Call. Aet. 1 fr. 1 und 2 Pfeiffer vgl. Kambylis (1965) 70.

65 Das wichtigste Zeugnis findet sich in den Florentiner Scholien zu Call. Aet. 1 fr. 1 und 2 Pfeiffer, wo es heisst, Kallimachos sei, als er im Traum den Musen auf dem Helikon begegnete,

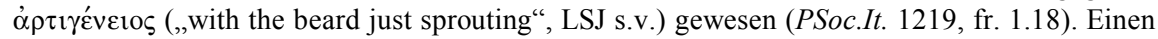
indirekten Hinweis gibt Kallimachos selber (fr. 1.37-38 Pfeiffer), indem er betont, dass die Musen diejenigen, welche sie schon als Kind $(\pi \alpha \hat{\imath} \delta \alpha \varsigma)$ angeschaut haben, auch im Alter nicht fallen lassen.

66 Vgl. insbesondere Call. Aet. 1 fr. 1.23-28 Pfeiffer (= 1.23-28 Asper; Apollon spricht): ........ ...

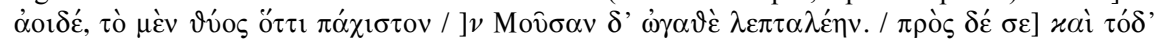

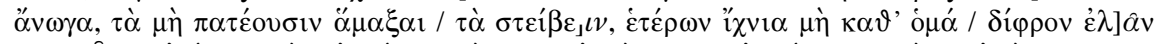

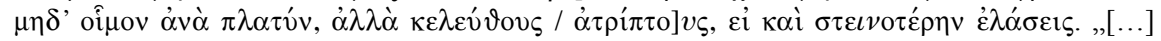
Sänger, das Opfer möglichst fett [...] aber die Muse, mein Bester, dünn! [Ausserdem] trage ich dir auch auf, was Wagen nicht befahren, das zu betreten, in den Spuren anderer nicht [den Wagen zu steuern] noch auf breiter Strasse, sondern unberührte Wege, magst du auch einen engeren fahren!“ - Textgrundlage und Übersetzung: Asper (2004) 66-69.

67 Hesiod wird bei Kallimachos namentlich erwähnt (fr. 2.2 Pfeiffer). Die Entrückung auf den Helikon ist uns in $A P 7.42 .5$ sowie in den Florentiner Scholien (PSoc.It. 1219, fr. 1.1-2) überliefert.

68 Zu Kallimachos' Aitienprolog im Gesamten vgl. Kambylis (1965) 69-123. 
Vorgänger auseinandersetzt. ${ }^{69}$ Quintus knüpft nun mit einer ganz bestimmten Wendung einen intertextuellen Faden sowohl zu Hesiod als auch zu Kallimachos:

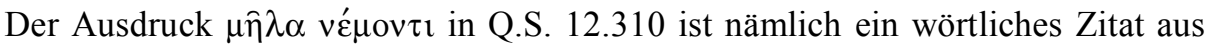
Kallimachos' Musenweihe und bezieht sich bei jenem auf Hesiod:

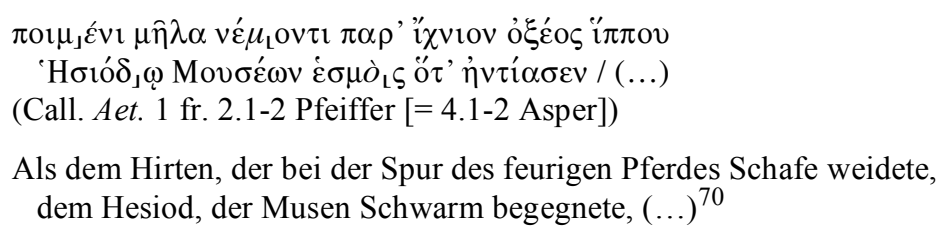

Quintus zitiert also aus einem hellenistischen Text, welcher seinerseits bereits auf einen anderen, archaischen Text referiert, auf welchen Quintus hinwiederum auch Bezug genommen hat.

(3) Die Tatsache, dass es sich um eine Traumerzählung handelt, ist zwar ebenfalls nicht direkt aus Kallimachos' Fragmenten ersichtlich, ist aber in späterer Überlieferung gut genug bezeugt, um als sicher gelten zu können. ${ }^{71}$ In Th. 2728 wird zum erstenmal in der abendländischen Dichtung mit den Versen ` $\delta \mu \varepsilon v$

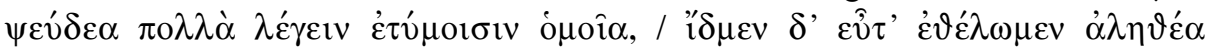
үฺ Wahrheit' gestellt - die Verse stellen, freilich im Gewand mythischer Rede, die erstmals fassbare Reflexion über das literaturtheoretische Grundproblem schlechthin dar. Kallimachos seinerseits rührt ebenfalls an diese Thematik, sowohl mittels seiner Bezugnahme auf die hesiodeische Vorlage als auch allgemein durch das Setting in einem Traum, also in einem Schwebezustand zwischen zwei Welten. Mit seinem Rückgriff auf Verse aus diesen beiden kanonischen Vorlagen referiert Quintus also auf Kontexte, deren Hauptthema letztlich die Frage nach ,Dichtung und Wahrheit' ist. $^{72}$ Liest bzw. denkt man diese Assoziati-

69 Vgl. Kambylis (1965) 122: „Kallimachos hat das Motiv der Dichterweihe von Hesiodos übernommen, es aber neu gestaltet. Dazu haben Ansätze beigetragen, die er bei seinem Vorbild vorfand; das Erlebnis des Hesiodos ist bei ihm zu einem Traum geworden, das Wasser wird zu einem Symbol für die Dichterweihe. Sowohl die Übernahme des Motivs (und somit Kallimachos' ausdrückliche und unmittelbare Berufung auf sein Vorbild) als auch seine Verwendung überhaupt und - verbunden damit - die Notwendigkeit, es weiter auszugestalten, erwuchsen aus der besonderen geistigen Situation der hellenistischen Zeit.“

70 Textgrundlage und Übersetzung: Asper (2004) 70-71.

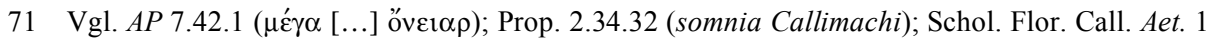
fr. 2 Pfeiffer (PSoc.It. 1219, fr. 1.16: $\kappa] \alpha \tau^{\prime}$ 'óv $\left.\alpha \rho\right)$.

72 In diesem Zusammenhang dürfte auch die Aufforderung, die Männer, welche das Pferd bestei-

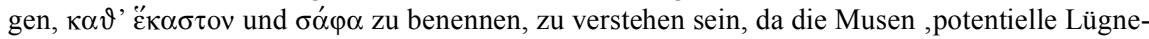
rinnen' sind und da gerade an dieser Stelle genaue und korrekte Angaben über die betreffenden Helden zwingend notwendig sind. 
onen als ,intertextuelle Tonspuren' bei der Lektüre unseres Musenanrufs mit, so wird einem, wie mir scheint, nicht zuletzt nahegelegt, dessen Wahrheitsgehalt bzw. autobiographischen Wert kritisch zu betrachten - eine Frage, welche, wie unten in Kap. 4. noch zu zeigen sein wird, in Quintus' Musenanruf von eminenter Wichtigkeit ist.

Der Hauptzweck der intertextuellen Bezüge zu Kallimachos' Aitienprolog dürfte demnach ein poetologischer sein und darin bestehen, dass Quintus damit seine Positionierung als poeta doctus bzw. als Homerus novus umreisst, der die alte, traditionelle Epik mit den ,Vorzügen' der alexandrinischen Kleinpoesie anreichert und somit etwas im Bereich des Epos Originäres generieren will. Diese Methode der doppelten Bezugnahme sowohl auf die archaische Epik als auch auf die hellenistische Dichtung - wobei ersterer klar die Hauptrolle und letzterer eher die Funktion eines ,Supplements' zukommt - ist typisch für Quintus' Posthomerica und lässt sich auch andernorts beobachten. ${ }^{73}$ Das Raffinierte dabei ist, dass Quintus auf eine bereits existierende intertextuelle Verknüpfung zurückgreift, dass er also nicht etwas grundlegend Neues kreiert, sondern aufbauend auf schon Bestehendem etwas Eigenes erschafft und somit den Faden der intertextuellen Beziehungen weiterspinnt. Durch die Bezugnahme auf Kallimachos referiert Quintus zudem auf ein Dichtungsideal, welches seinerseits schon den Anspruch hatte, auf der Basis von Bestehendem etwas Neues zu schaffen, womit er sich nicht zuletzt auch in die hellenistisch-alexandrinische Tradition einschreibt. $\mathrm{Zu}$ fragen wäre hierbei freilich, was es zu bedeuten hat, dass Quintus auf ein Vorbild rekurriert, welches der traditionellen epischen Dichtung derart kritisch gegenübersteht. Damit verbunden ist ein altes Problem der Kallimachosforschung, nämlich ob die quantifizierenden Antithesen (z.B. gross-klein; dick-dünn; schmal-breit) in Kallimachos' poetologischem Programm , eigentlich' oder ,uneigentlich` zu verstehen seien, d.h. ob der Dichter damit konkret die Länge/

73 Ein Beispiel möge genügen: In Q.S. 1.403-476 treffen zwei Trojanerinnen in Rede und Gegenrede aufeinander. Sie diskutieren, inspiriert durch die Amazonen, die Frage, ob Frauen grundsätzlich auch zum Krieg befähigt seien und sie deshalb ihren Männern zu Hilfe eilen sollten oder ob dies allein Sache der Männer sei. Prinzipielles Vorbild dieser Episode sind homerische Beratungsreden wie etwa die im 2. Buch der Ilias. Gleichzeitig jedoch klingt m.E. eine Szene aus Apollonios' Argonautika an, nämlich die Beratung der Lemnierinnen über die Frage, ob sie die Argonauten, die mit ihrem Schiff an der Küste ihrer Insel gestrandet sind, in die Stadt einlassen sollen (A.R. 1.633-708). Diese Szene ist enorm komisch, da es den Lemnierinnen evidentermassen nur darum geht, endlich wieder einmal sexuelle Befriedigung zu erfahren, nachdem sie ihre eigenen Männer aus Eifersucht alle getötet haben. Hält man sich bei der Lektüre von Q.S. 1.403-476 auch diese Episode vor Augen (obschon sie freilich nicht das Hauptvorbild ist), so bekommt die Diskussion der Trojanerinnen eine ironische Färbung, insofern als ihre objektive Nichtigkeit und Lächerlichkeit offensichtlich wird. Das Intermezzo endet denn auch damit, dass die Frauen auf die zweite Rednerin hören und bei ihren Wollarbeiten bleiben. 
Grösse bzw. Kürze eines Werks oder vielmehr metaphorisch den Stil meine. ${ }^{74}$ Quintus jedenfalls muss Kallimachos' Poetik mit Sicherheit stilmetaphorisch gelesen haben, denn andernfalls würde er sein ganzes Tun als homerisierender Epiker geradezu ironisieren. ${ }^{75}$

Mit dem Adjektiv $\pi \varepsilon \rho ı \kappa \lambda v \tau \alpha ́$ stellt Quintus gleichsam ein ,Hinweisschild“ auf, welches auf die genannte doppelte intertextuelle Verbindung zu Hesiod und Kallimachos aufmerksam machen soll: An der Wortoberfläche lässt sich die

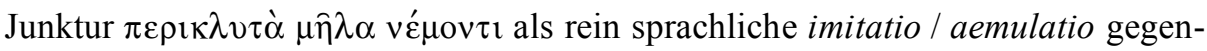
über Homer bzw. als kumulative Verknüpfung homerischen, hesiodeischen und kallimacheischen Ausdrucks verstehen: In Od. 9.308 ist nämlich von Polyphems Schafen als $\kappa \lambda v \tau \grave{\alpha} \mu \hat{\eta} \lambda \alpha$ (,das herrliche Vieh") ${ }^{76}$ die Rede; indem Quintus die Kollokation aufgreift und mittels der Vorsilbe $\pi \varepsilon \rho 1$ - variiert, reichert er die hesiodeisch-kallimacheische Stelle mit einem zusätzlichen homerischen Chroma an. Wichtiger hingegen scheint mir eine inhaltliche Implikation zu sein: Das komponierte $\pi \varepsilon \rho \imath \kappa \lambda \nu \tau$ ó $\varsigma$ wird bei Homer u.a. als Epitheton für die beiden odysseischen Sänger Phemios und Demodokos verwendet, ${ }^{77}$ ist also a domo ein mit der Poesie verknüpftes Wort und muss somit hier auf die hesiodeisch-kallimacheische Vorlage gemünzt sein, muss also im Sinne einer , alexandrinischen Fussnote ",das «von Hesiod und Kallimachos her〉 berühmte Vieh“ meinen. Dem Leser wird dadurch eine poetologisch-intertextuelle und somit betont unkonkrete, unbiographistische Lesart des ganzen Binnenproömiums geradezu aufoktroyiert. ${ }^{78}$

74 Vgl. Asper (1997) 238: „Im Hinblick auf Kallimachos stellt sich die Frage, ob in den Metaphern, die Quantitätsbegriffe verwenden, diese Quantitäten selbst noch metaphorisch aufzufassen sind oder bereits zum tenor gehören. Wenn z.B. von der „grossen Flut" des assyrischen Flusses die Rede ist, meint Kallimachos damit etwas real ,Grosses‘ (etwa ein langes Gedicht) oder steht ,gross' als Metapher für etwas anderes? Auf dieser Ambivalenz beruht die alte Unsicherheit der Kallimachos-Forschung, ob es diesem in seiner Polemik essentiell um den Gegensatz von langen und kurzen Gedichten gehe oder nicht.“

75 Gemäss Asper (1997) sind die Quantitätsbegriffe auch bei Kallimachos selber auf einer uneigentlichen, stilmetaphorischen Ebene zu lesen (239): „Kallimachos lässt zwar die Telchinenpolemik von realen Grössenbegriffen aus ihren Vorwurf lancieren, doch nützt er die offensichtliche Absurdität dieser Polemik [...] für seinen metaphorischen Gegenangriff aus. Aus realen Quantitäten werden quantifizierende Metaphern.“

76 Zur Bedeutung vgl. Campbell (1981) 104: „,[T]he sheep are ,renowned', highly thought of, i.e.

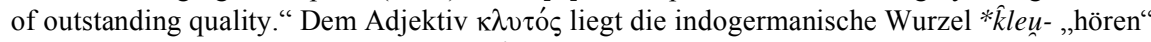
zugrunde, wovon auch das Substantiv $\kappa \lambda \dot{\varepsilon}$ o $\zeta$ abgeleitet ist.

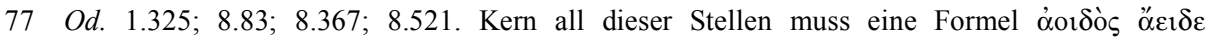
$\pi \varepsilon \rho \iota \kappa \lambda v \tau$ ó (,„es sang der weitberühmte Sänger“) sein (mit auffälliger figura etymologica).

78 Bezeichnenderweise konnten die alten Interpreten mit $\pi \varepsilon \rho \imath \kappa \lambda v \tau \alpha \dot{~ n i c h t s ~ a n f a n g e n ~ u n d ~ h a b e n ~ e s ~}$ als reines epitheton ornans aufgefasst; vgl. Tychsen (1783) 6: „Est hoc merum epitheton poeticum, $[\ldots]$ nihil amplius. “ 


\section{Ein Schäfer „,in Smyrnas Landen“: Biographismus vs. Poetologie}

\subsection{Smyrna als Geburtsstadt Homers und ,Hauptstadt ${ }^{\star}$ der Zweiten Sophistik}

Quintus wird, soweit uns bekannt ist, zum ersten Mal von Johannes Tzetzes (1110-1180) als ó $\Sigma \mu \nu \rho v \alpha i o \varsigma$ bezeichnet ${ }^{79}$ - die Verortung unseres Dichters in Smyrna ist also, zumindest gemäss unserem Wissensstand, nicht antik. Dass Tzetzes das Toponym aus dem Musenanruf im 12. Buch hergeleitet hat, ist anzunehmen. Konstantin Laskaris (1434-1501) sodann geht im Vorwort seiner Abschrift des verloren gegangenen Hydruntinus $(\mathrm{H})^{80}$ von Quintus' Herkunft aus Smyrna aufgrund von Q.S. 12.310 explizit aus. ${ }^{81}$ Die autobiographische Aussagekraft des Passus wurde in den folgenden Jahrhunderten grundsätzlich nicht in Frage gestellt - so konstatiert etwa Tychsen nach einer kritischen Besprechung des Binnenproömiums unmissverständlich, man könne, aufgrund dieser Stelle auf jeden Fall darauf schliessen, dass Smyrna die Heimat unseres Dichters gewesen sei $^{`} .82$ Allerdings konkurrierte die Bezeichnung Smyrnaeus zuweilen mit Calaber, wofür der Fundort des von Basilius Bessarion (1403-1472) zwischen 1453 und 1462 im Kloster von San Niccolò di Casoli in Apulien entdeckten Hydruntinus ausschlaggebend war, dessen Region - Apulien wurde in der Antike Calabria genannt - man kurzerhand auch als Heimat des Dichters ansah. ${ }^{83}$

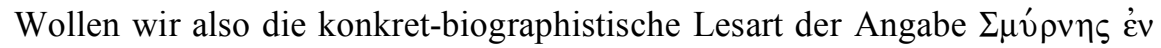
$\delta \alpha \pi \varepsilon ́ \delta 01 \sigma \iota$ in Frage stellen, so hören wir in der Tat einige gewichtige Riesen hinter uns hermarschieren. Gleichwohl lassen sich gewichtige Argumente anbringen. Zwei Aspekte sind zu berücksichtigen:

79 Vgl. Vian (1963) VII Anm. 2 (mit Stellenangaben). Tzetzes verwendet Quintus' Epos als Quelle für seine eigenen Posthomerica und nimmt mehrfach explizit auf ihn Bezug; vgl. Vian a.a.O. Anm. 1 (mit Stellenangaben).

80 Matritensis gr. $4686(=q)$. Zu Laskaris' Editionsprojekt von 1496 vgl. Vian (1959b) 32-38.

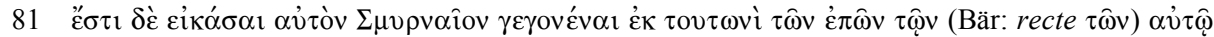

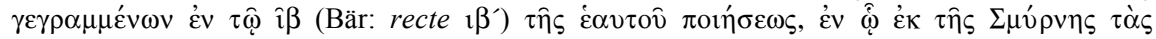

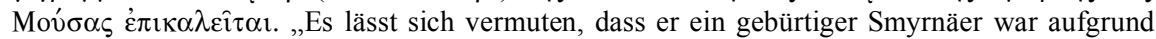
der folgenden Worte, die er selber in Buch 12 seines Gedichts schreibt, worin er die Musen aus Smyrna anruft." (Es folgt ein Zitat von Q.S. 12.306-313.) Laskaris' Vorwort ist in voller Länge abgedruckt bei Köchly (1850) CXI (Zitat danach).

82 Tychsen (1807) XXV: „Hoc igitur saltem ex isto loco licet colligere, Smyrnam fuisse patriam nostri.“

83 Der Erstdruck der Aldina (1505) wurde unter dem Titel Quinti Calabri derelictorum ab Homero libri XIV publiziert. Bereits Rhodomann jedoch hat in der praefatio seiner Ausgabe von 1604 klar erkannt, dass Calabria nur der (zufällige) Fundort, nicht jedoch die Heimat des Dichters sein konnte; gleichwohl hat er (möglicherweise auf Wunsch oder Druck des Verlegers?) seine

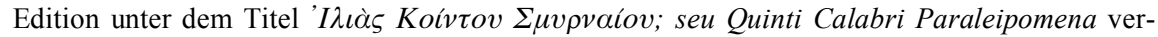
öffentlicht. Die Bezeichnung Quintus Calaber blieb sodann bis ins 19. Jh. durchaus geläufig. Vgl. Vian (1963) VIII; James / Lee (2000) 3; James (2004) XXI. 
(1) Smyrna gehörte in der Antike zu den nicht wenigen Städten, die für sich die Ehre, Homers Heimatstadt zu sein, in Anspruch nahmen. Verschiedene literarische Quellen legen beredtes Zeugnis dafür ab, dass Smyrna in diesem ,Kampf der Städte ${ }^{84}$ eine prädominante Stellung einnahm, so etwa die kaiserzeitlichen Homer-Biographien oder eine Reihe von Epigrammen. ${ }^{85}$ Berühmt ist ein dem Antipatros zugeschriebener Zweizeiler, welcher unter sieben Städten, die sich um Homers Geburt zanken, auch Smyrna nennt; in einem anderen Epigramm der Anthologia Palatina ist von Smyrna als „Stadt Homers“ mit aller Selbstverständlichkeit die Rede. ${ }^{86}$ Im Certamen Homeri et Hesiodi sodann wird Smyrna von allen potentiellen Heimatstädten Homers an erster Stelle genannt, ${ }^{87}$ und im spätantiken christlichen Epos Iohannis des Flavius Cresconius Corippus (5. Jh. n. Chr.) wird Homer als Smyrnaeus vates bezeichnet. ${ }^{88}$ Auch im engeren Kontext der Zweiten Sophistik - z.B. bei Autoren wie Aelius Aristides oder Dio Chryso-

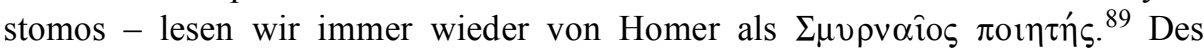
Weiteren wusste man von einem 'Oún Tempel in Smyrna in der Nähe des Flusses Meles zu berichten. ${ }^{90}$ Kurzum: Smyrna war nicht einfach einer von den vielen Orten, welche als Heimat Homers galten oder gelten wollten, sondern der Stadt wurde, aller Konkurrenz zum Trotz, dieser Rang grundsätzlich unwidersprochen zuerkannt ${ }^{91}$ - eine Auffassung, die

84 Zur antiken Frage nach ,Homers Heimat' und zum daraus resultierenden ,Streit der Städte 'vgl. Wilamowitz (1916) 367-373; Skiadas (1965) 18-32; Latacz ( $\left.{ }^{4} 2003\right)$ 33-34.

85 Vgl. Ps.-Hdt. Vit.Hom. 19-21; Ps.-Plu. Vit.Hom. 17-20; AP 9.672; 11.442; 16.295; 16.296 (Antip.Sid.); 16.297; 16.298; 16.320.

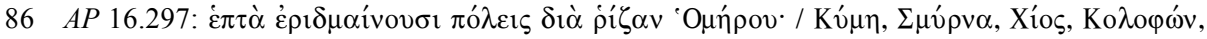

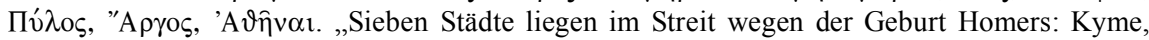

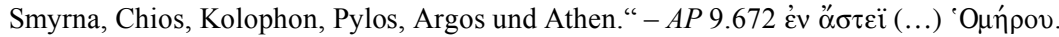

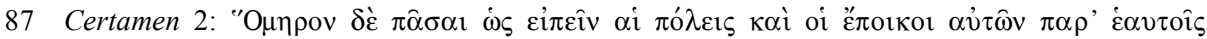

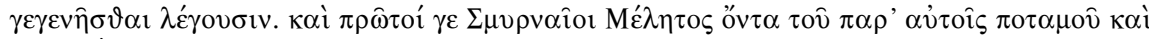

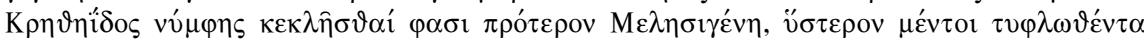

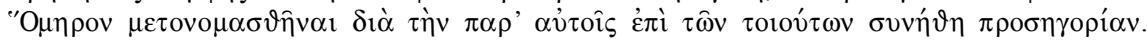
„Sozusagen alle Städte und deren Einwohner behaupten, dass Homer bei ihnen geboren sei. Und in erster Linie sagen die Smyrnäer, dass er früher ,der Meles-Geborene' hiess, weil er vom Fluss Meles, der sich bei ihnen 〈befindet〉, und von der Nymphe Kretheïs abstammte; später dagegen sei er wegen seiner Erblindung in "Homer' umgetauft worden aufgrund der bei ihnen dafür gebräuchlichen Bezeichnung."

88 Coripp. praef. 11-12: Smyrnaeus vates fortem descripsit Achillem, / Aeneam doctus carmine Vergilius. „Der Sänger aus Smyrna beschrieb den tapferen Achilles, und den Aeneas in seinem Gedicht der gelehrte Vergil.“

89 Vgl. z.B. Aristid. Or. 17.8; 17.15; 21.8; 23.21; 33.29; D.Chr. 7.119; Paus. 7.5.12.

90 Vgl. Str. 14.1.37. - In der Tat ist in der Antike häufig bezeugt, dass Homer am Fluss Meles,

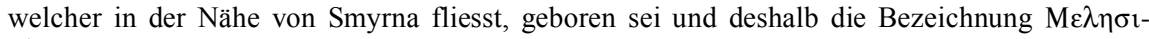

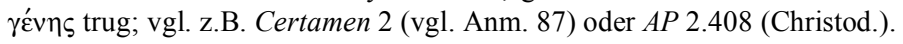

91 Vgl. Skiadas (1965) 25 Anm. 3: „Immerhin wagte fast niemand die Geburt in Smyrna zu bezweifeln $[\ldots]$, und alle Städte versuchten, sich mit Smyrna irgendwie zu verbinden.“ 
auch die positivistische Quellenforschung des 19. Jahrhunderts noch weitgehend vertrat und etwa Ulrich von Wilamowitz-Möllendorff ,an der Existenz eines

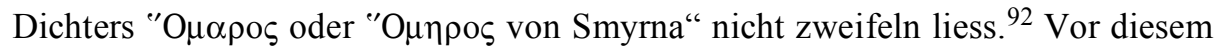
Hintergrund besehen, ist eine unkonkrete, poetologische Lesart von Quintus'

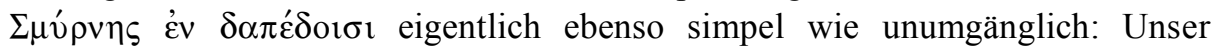
Dichter - bzw., genauer, das epische ,Ich', der implizite Autor - stellt sich mithilfe dieser Lokalisierung als ,zweiter Homer' vor, so wie er sich im Zusammenhang mit der Musenweihe schon als ,zweiter Hesiod‘ bzw. als ,zweiter Kallimachos' geriert hat.

(2) Danebst ist es m.E. auch vorstellbar, dass Quintus mit der Erwähnung Smyrnas sich nicht nur in die literarische Tradition von Homers Geburtsstadt, sondern zugleich auch ins Umfeld der Zweiten Sophistik einschreibt, da Smyrna ein bedeutendes kulturelles Zentrum, ein melting pot, sozusagen die ,Hauptstadt ${ }^{\text {‘ }}$ jener intellektuellen Strömung im zweiten und dritten Jh. n. Chr. war. ${ }^{93}$ Auf einer

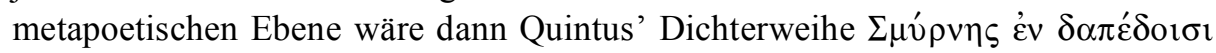
als eine Verortung unseres Dichters nicht bloss in der homerisch-epischen Tradition, sondern auch im zeitgenössischen Kontext der Zweiten Sophistik aufzufassen.

Während eine Verbindung des Quintus zu Smyrna unter dem Aspekt der Zweiten Sophistik m.W. bis anhin noch nicht erwogen wurde, hat man die poetologische Implikation des Städtenamens als Geburtsort Homers längst bemerkt und meist auch grundsätzlich gutgeheissen. Trotzdem wird in der Regel an der autobiographischen Aussagekraft des Passus festgehalten, wofür zwei Argumente ins Feld geführt werden: erstens die Detailliertheit und Genauigkeit der ganzen Beschreibung in Q.S. 12.310-313, was gleichwohl eine konkrete Lokalisierung wahrscheinlich mache, ${ }^{94}$ und zweitens die Tatsache, dass Quintus an anderen

92 Wilamowitz (1916) 372. - Desgleichen auch in neuerer Zeit noch Gauer (1996), welcher gar glaubt, in ,Homers' Beschreibungen Trojas das antike Smyrna und somit die Heimat des Dichters wiedererkennen zu können.

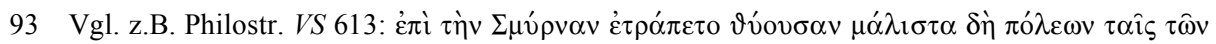

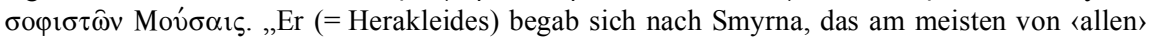
Städten den Musen der Sophisten opferte." - Vgl. ferner (exempli gratia) Philostr. VS 516; 518; $531 ; 582$.

94 Vgl. beispielsweise James (2004) XVIII: „Quintus' location at Smyrna does not have the appearance of a purely literary element, even though Smyrna was famous for its claim to be the birthplace of Homer. The insistent particularity of the topographic details would seem to lack point other than as a factual record. They cannot be verified, but they are at least compatible with the territory of Smyrna between the river Hermos and Mount Sipylos." - Vgl. auch Vian (1963) IX-XIII und James / Lee (2000) 4. 
Stellen der Posthomerica mehrfach gute Kenntnisse kleinasiatischer Landschaften beweise, was auf eine entsprechende Herkunft schliessen lasse. ${ }^{95}$

Das zweitgenannte Argument lässt sich m.E. leicht entkräften: Ausführliche topographische Beschreibungen u.dgl. müssen nicht zwingend das Resultat von Autopsie und schon gar nicht von Ortsansässigkeit oder Abstammung bedeuten, da entsprechende Informationen entweder auf eigenen Reisen oder aber auch nur aus den in der Antike reichhaltig vorhandenen schriftlichen Reisebeschreibungen anderer ${ }^{96}$ erworben bzw. bezogen werden konnten. ${ }^{97}$ Man denke nur daran, wie lange sich die positivistische Quellenkritik unnötig den Kopf zerbrochen hat über den empfundenen Widerspruch zwischen Homers angeblicher Blindheit einerseits und der Fülle und Anschaulichkeit seiner Beschreibungen andererseits. ${ }^{98}$

Das erstgenannte pro-biographistische Argument - die Detailgetreue der Ortsbeschreibung - bedarf dagegen einer eingehenderen Prüfung. Ich möchte deshalb im Folgenden zu zeigen versuchen, dass sich auch der Rest des Proömiums, d.h. namentlich die topographische Beschreibung in Q.S. 12.310-313, in (fast) allen Einzelheiten auf einer poetologischen Ebene lesen und somit in das bisher Gesagte einreihen lässt und dass sich die ,genaue Beschreibung' der Topographie bei näherem Besehen als etwas ganz Anderes entpuppt.

\subsection{Artemis und die drei genera dicendi}

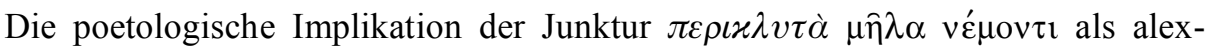
andrinische Fussnote wurde bereits erläutert (s.o. Kap. 3.3.). Da, wie ausgeführt, das Adjektiv $\pi \varepsilon \rho \imath \kappa \lambda \nu \tau \alpha ́$ im Kontext der zugrunde liegenden kanonischen Subtexte eine poetologische Lesart nachgerade aufzwingt, wird man sich unweigerlich

$95 \mathrm{Zu}$ denken wäre beispielsweise an Q.S. 1.291-306 - eine Digression, die eine anschauliche Beschreibung des Sipylos-Gebirges bietet. Vgl. James (2004) XVIII: „A further consideration is that the authenticity of Quintus' Smyrnaean background receives general support from the quite numerous passages in his epic that show detailed knowledge of western Asia Minor."

96 Man denke etwa an die zahlreichen Digressionen in Herodots Historien oder an die $\pi \varepsilon \rho ı \eta \eta \dot{\sigma} \sigma \varepsilon 1 \varsigma$ des Herakleides oder des Pausanias. Vgl. auch Walde (2001).

97 In diesem Sinne schon Vian (1959a) 143: „Ce ne sont pourtant pas des impressions personnelles que livrent les Posthomerica, mais plutôt des souvenirs de lecture [...]“- Ein hübsches Beispiel aus der Zeit der Zweiten Sophistik mag dies illustrieren: Philostrat berichtet in seinen Sophistenviten über Aelian, dieser habe sein Lebtag Italien nie verlassen und nie seinen Fuss auf ein Schiff gesetzt (Philostr. VS 625). Aelian seinerseits jedoch bietet in seiner Varia historia eine akkurate Beschreibung des thessalischen Tempe-Tals (Ael. VH 3.1), welches er, sofern wir Philostrats Bericht Glauben schenken wollen, nie mit eigenen Augen gesehen hat.

98 Vgl. beispielsweise Wilamowitz (1916) 421: „Sieht man [...], dass er seine Gedichte aufschreibt, eine schöne Pinie besingt, den Eindruck einer erleuchteten Halle schildert, so drängt sich der Schluss auf, dass dieses ganze Leben von einem armen Rhapsoden erzählt worden ist, aber von keinem blinden $[\ldots]^{“}$ 
fragen müssen, wie es um den Rest des Proömiums (Q.S. 12.311-313) steht. Dass der Städtename Smyrna als potentielle Geburtsstadt Homers und möglicherweise auch als kulturelles Zentrum der Zweiten Sophistik (auch) in diese Kategorie eingeordnet werden kann (oder muss), haben wir gesehen. Einen Anhaltspunkt für eine autonome, unkonkrete Deutung des Flussnamens Hermos scheint es mir dahingegen nicht zu geben; hier muss wohl der Hinweis genügen, dass dieser Fluss, den Quintus gesamthaft zweimal erwähnt, ${ }^{99}$ der Hauptfluss der Gegend um Smyrna ist und also von der Interpretation des Städtenamens mitbetroffen ist. ${ }^{100}$ Eine grössere Schwierigkeit dürfte die Frage nach der Bedeutung von Artemis in einem Zusammenhang wie diesem sein. Abgesehen vielleicht von ihrer geschwisterlichen Beziehung zu Apollon, hat diese Göttin wahrhaftig wenig mit der Musenkunst zu schaffen. Warum aber heisst es dann, das epische ,Ich` habe

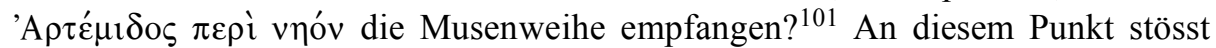
die postulierte poetologische Deutung von Quintus' Musenanruf wohl an eine Grenze. Gleichwohl wird ihre grundsätzliche Plausibilität deswegen nicht beeinträchtigt: Bedenkt man nämlich, dass Artemis in Kleinasien eine überaus wichtige und präsente Gottheit darstellte, ${ }^{102}$ so muss man eingestehen, dass eine Angabe

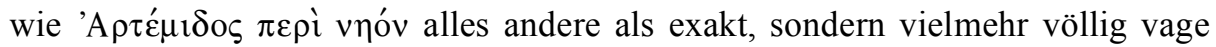
ist. ${ }^{103}$ Dem vermögen auch die restlichen ,genauen Beschreibungen' kaum abzuhelfen, da der Ausgangspunkt für eine exakte Lokalisierung, eben der genannte Artemistempel, gar nicht lokalisierbar ist. Dieser ergibt vielmehr zusammen mit dem Fluss Hermos ein in sich stimmiges Gesamtbild, welches zu Smyrna und dessen Umgebung passt und somit den Musenanruf auch auf einer konkreten Ebene kohärent lesbar macht. Indes bringt es die Tatsache, dass ein Artemistempel „in Smyrnas Landen“ sich vielerorts befinden könnte und somit ,utopisch“ im eigentlichen Wortsinn ist, mit sich, dass eine konkrete Lokalisierung der ganzen Szenerie gar nicht möglich ist. Dies jedoch darf wiederum als Hinweis darauf verstanden werden, dass man als Leser nicht bei einer konkreten Lesart bleiben soll, sondern dass man die poetologische Interpretationsebene einbeziehen muss - ja, man könnte möglicherweise so weit gehen, die Vagheit der Topo-

99 Hier sowie in Q.S. 1.296.

100 Der Fluss Hermos wird schon in der frühesten Dichtung (Il. 20.392; Hes. Th. 343) und später verschiedentlich in geographischen Schriften oder Exkursen erwähnt; vgl. Kaletsch (1998).

101 Nicht zuletzt diese Unklarheit dürfte mitunter mit ein Grund sein, weshalb man in der Regel geneigt ist, trotz der bemerkten poetologischen Implikation des Städtenamens Smyrna gleichwohl die ganze topographische Beschreibung als autobiographischen Passus zu lesen.

$102 \mathrm{Zu}$ den verschiedenen Artemiskulten im kleinasiatischen Raum vgl. Schreiber (1884-1890) 588594; Burkert (1977) 233. Zu Artemis im Allgemeinen vgl. Graf (1997); ferner Petrovic (2007).

103 Es handelt sich also keineswegs, wie Koster (1970) 156 es formuliert, um eine „übergenaue[n] Lokalangabe“, mit welcher „die Glaubwürdigkeit der inspirierten Dichtung abgesichert werden musste“. Auch die Erwähnung des Flusses Hermos, des Hauptflusses von Smyrna (vgl. Anm. 100 ), trägt zur Genauigkeit nichts bei. 
graphie auf einer Metaebene als Sinnbild für die nebulöse, schemenhafte Gestalt des Dichters Quintus zu lesen, welcher dadurch als ,zweiter Homer' umso authentischer wirkt, da Homer seinerseits ja auch eine äusserst schattenhafte Erscheinung darstellt. So oder so besteht m.E. der Irrtum bisheriger Interpreten darin, im Zusammenhang mit den vorliegenden topographischen Beschreibungen Vagheit für Genauigkeit gehalten und daraus auf die Notwendigkeit einer biographischen Interpretation geschlossen zu haben. ${ }^{104}$

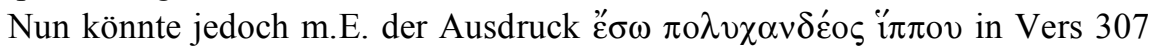
einen möglichen Anhaltspunkt liefern, die Göttin Artemis trotz allem in eine poetologische Gesamtdeutung einzubinden: Das Adjektiv $\pi \mathrm{o} \lambda \nu \chi \alpha \nu \delta \dot{\prime} \varsigma$,vielfassend" scheint ein alexandrinischer Neologismus zu sein (Erstbeleg bei Theoc. Id. 13.46) und ist vor Quintus nur vereinzelt fassbar. ${ }^{105}$ Dieser sodann ist der erste, der es insgesamt achtmal und somit in noch nie dagewesener Dichte verwendet. ${ }^{106}$ Triphiodor, dessen zeitliches Verhältnis zu Quintus nach wie vor strittig ist, ${ }^{107}$ gebraucht das Wort zweimal, und zwar auffälligerweise einmal

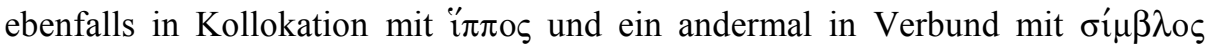
(,Bienenstock“), dort allerdings in einem Vergleich, welcher sich auch auf das hölzerne Pferd bezieht. ${ }^{108}$ Später ist das Wort nebst einigen prosaischen Belegen vor allem bei Nonnos wieder anzutreffen (gesamthaft 14 Belege). ${ }^{109}$ Unser Dichter verwendet das Adjektiv in verschiedenen Kontexten bzw. Kollokationen, d.h. in Verbindung mit unterschiedlichen Substantiven, und bindet es - im Gegensatz

104 Zwar ist ein Artemistempel in Smyrna archäologisch nicht nachzuweisen, doch wird die Göttin inschriftlich mehrfach erwähnt (Hinweis von Prof. Dr. Walter Burkert). Dies kann meine These von der ,Utopie“ des Ortes nur stützen: Ein Artemistempel „in Smyrnas Landen“ kann nirgends und überall sein.

105 Dichterische Belege, die mit Sicherheit vor Q.S. anzusetzen sind: Theoc. Id. 13.46; Nic. Ther. 951; Opp. H. 5.331.

106 Belege bei Q.S.: 1.527; 2.136; 3.731; 4.475; 9.390; 12.264; 12.307; 13.138. - Freilich ist zu bedenken, dass eine Dichte von acht Belegen für Quintus, der gewisse Wörter mit nachgerade penetranter Exzessivität verwendet, alles andere als viel ist; gleichwohl ist der sprunghafte Anstieg beachtenswert.

107 Triphiodor wird tendenziell als der jüngere angesehen; vgl. dazu Dubielzig (1996) 11, mit einer vollständigen Liste der diesbezüglichen Forschungsliteratur. Vgl. auch James / Lee (2000) 5: „Stylistic links between Triphiodorus and Nonnus make it probable, though not certain, that Q. antedates them both."

108 Triph. 412 (Kassandra warnt vergeblich vor dem unheilbringenden Danaergeschenk):

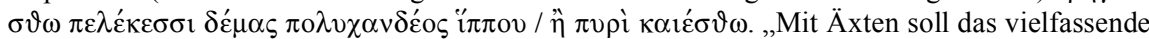
Pferd zerschlagen oder mit Feuer verbrannt werden!“ - Triph. 533-541: Die griechischen Helden steigen nachts aus dem Pferd und stürzen sich mordend auf die schlafenden Trojaner; dabei werden sie mit Bienen verglichen, welche ,im vielfassenden Bienenstock“ (535 $\pi \mathrm{o} \lambda \nu \chi \alpha \nu \delta \delta$ ́́

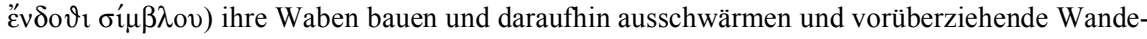
rer stechen - der Bienenstock dient also als Metapher für das hölzerne Ross.

109 Belege bei Nonnos: D. 2.441; 11.162; 15.19; 16.257; 18.284; 26.305; 34.252; 41.69; Par. Eu. Io. $6.51 ; 10.24 ; 12.8 ; 14.8 ; 18.77 ; 21.34$. 
$\mathrm{zu}$ anderen Wörtern - nicht in eine neu kreierte, homerisierende Formel ein. ${ }^{110}$ Letztere Beobachtung ist insofern von Belang, als da sie eine gezielte Deutung

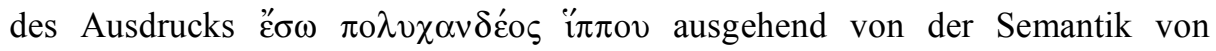
$\pi \circ \lambda v \chi \alpha v \delta \dot{\eta} \varsigma$ rechtfertigt, da keinerlei formelhafte und somit auch keine stehende, semantisch abgeschwächte Verwendung des Adjektivs im Sinne eines epitheton ornans vorliegt. In Bezug auf das hölzerne Ross gebraucht Quintus das Wort

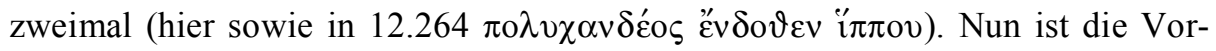
stellung vom trojanischen Pferd als, Mutter des Unheils' eine in der Antike weit verbreitete Metapher. ${ }^{11}$ Quintus evoziert mithilfe des Adjektivs $\pi \mathrm{o} \lambda \nu \chi \alpha v \delta \dot{\zeta} \varsigma$ ebendiese metaphorische Bedeutung und verstärkt selbigen Eindruck noch dadurch, dass er das Wort an zwei anderen, früheren Stellen mit dem Substantiv

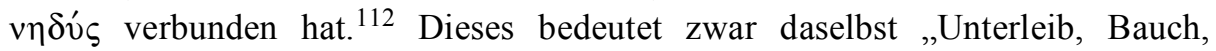
Magen“, doch dürfte die im Griechischen häufige Bedeutung „Mutterleib“113 gleichwohl mitschwingen. Das Adjektiv $\pi 0 \lambda v \chi \alpha v \delta \eta \dot{n} \varsigma$ ist somit in dem Moment,

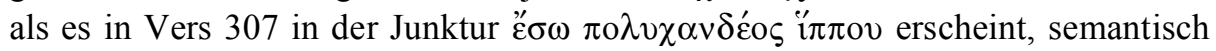
bereits so stark aufgeladen, dass sich der Gedanke an die Metapher vom , unheilschwangeren Pferd' wie von selbst ergibt. ${ }^{114}$ Vor diesem Hintergrund aber könnte sich m.E. auch die Erwähnung der Göttin Artemis in Vers 312 erhellen: Eine ihrer Funktionen ist die der Geburtsgöttin bzw. -helferin; zuweilen wird sie in der Antike auch mit Hekate oder Eileithyia assoziiert oder gar identifiziert. ${ }^{115}$ Der zeitgenössische Leser dürfte an jener Stelle, an welcher er die Geburtsmetapher vom ,unheilschwangeren Pferd' assoziierte, in Artemis möglicherweise die

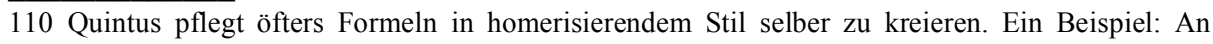
gesamthaft fünf Stellen verwendet er die vor ihm nicht belegte und also wohl von ihm selber er-

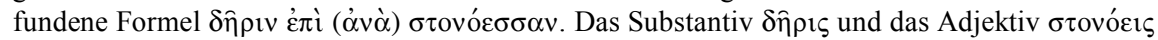
sind beides homerische Wörter, die jedoch kombiniert bei Homer noch nicht vorkommen und die Quintus auch einzeln exzessiv verwendet.

111 Vgl. Eur. Tr. 11; Lyc. 342; Enn. scen. 76; Verg. Aen. 2.20; 2.52; 2.238; AP 9.156 (Antiphil.); Triph. 389-390. - Vgl. auch den deutschen Ausdruck ,unheilschwanger“.

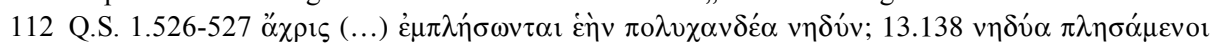
$\pi \mathrm{o} \lambda v \chi \alpha v \delta \dot{\varepsilon} \alpha$. In beiden Fällen handelt es sich um Tiervergleiche. Sprachliche Vorlage ist wohl Opp. H. 5.331, wo selbige Kollokation mit Bezug auf einen Wal erstmals vorkommt.

$113 \mathrm{Zu}$ dieser Bedeutung vgl. LSJ s.v. 4. - Den gemeinsamen Nenner zwischen ,Magen“ und ,Mutterleib“ stellt nicht nur die Ansiedlung im körperlichen Bereich ,um die Gürtellinie‘ dar, sondern auch die Grösse des ,Hohlraums'. In den beiden in Anm. 112 genannten Tiervergleichen geht es denn auch um wilde Tiere, welche ihren unersättlichen Appetit zu stillen suchen und ihren „vielfassenden Magen füllen“. Das Adjektiv $\pi \circ \lambda v \chi \alpha v \delta \eta \dot{\varsigma} \varsigma$ wird somit genau diesem tertium comparationis gerecht.

114 Die Aussage von Gärtner (2005) 174, dass „Quintus auf die Metapher des Krieger- bzw. Unheil,schwangeren“ Pferdes anders als Vergil und Triphiodor“ verzichte, müsste demnach modifiziert werden. Meines Erachtens lässt sich vielmehr die entsprechende Topik als Argument ins Feld führen, dass ein zeitgenössischer Leser ebenjene Assoziation in die Junktur ह้ $\sigma \omega \pi \mathrm{o} \lambda \nu \chi \alpha \nu \delta \varepsilon$ ć "i $\pi$ ov hineingelesen haben wird.

115 Vgl. dazu Schreiber (1884-1890) 571-573; Burkert (1977) 236; Petrovic (2007) 249-256. 
Geburtsgöttin gesehen haben. ${ }^{116}$ Da aber, wie wir gesehen haben, dem Leser eine poetologische Lesart des Musenanrufs mehrfach nahegelegt wird, schiene es doch wohl nicht abwegig, den Gedanken an die Geburtsgöttin und -helferin Artemis mit einer poetologischen Deutung zu verbinden: Artemis stünde, auf dieser Ebene gelesen, für den göttlichen Beistand bei der Kreation eines neuen Epos, eines neuen epischen Stils. Somit liesse sich - gestützt auf die durch das Adjektiv

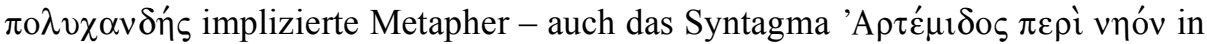
eine poetologische Lesart des ganzen Musenanrufs einpassen. ${ }^{117}$

Sodann bleibt nun noch der letzte Vers (Q.S. 12.313) zu klären. Hiefür hat Hopkinson eine nach meinem Dafürhalten plausible Deutung geliefert: Mit den

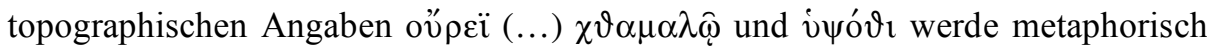
auf die beiden genera dicendi am äusseren Rand der drei Stile, d.h. auf das genus humile einerseits und auf das genus grande andererseits, Bezug genommen. ${ }^{118}$ Nebst den von Hopkinson angeführten, eher allgemein gehaltenen Begründungen vornehmlich sprachlich-stilistischer Natur ${ }^{119}$ lassen sich m.E. weitere Argumente dafür anbringen: Erstens scheinen die Ausdrücke an und für sich unzweideutig auf die Stilarten ${ }^{120}$ hinzuweisen: Der Begriff des $\dddot{\psi} \psi \circ \varsigma$ als Metapher für das genus grande ist in der Antike verbreitet. ${ }^{121}$ Das Adjektiv $\chi \vartheta \alpha \mu \alpha \lambda$ ó $\varsigma$ ist zwar als diesbezüglicher Gegenbegriff nicht nachgewiesen, lässt sich jedoch als antagonisti-

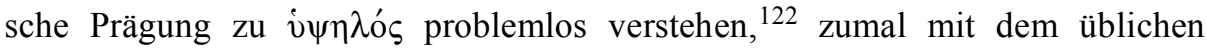
Terminus der i $\sigma \chi v o ́ \tau \eta \varsigma$ (wörtlich „Dürre, Trockenheit“) für das genus humile ja bereits eine metaphorische Ausdrucksweise geläufig war. ${ }^{123}$ Zweitens dünkt mich nicht unwesentlich, dass Quintus mit den Adverbien $\lambda_{i ́}^{\prime} v$ und $\pi \mathrm{o} \lambda \lambda \hat{\omega}$ einen

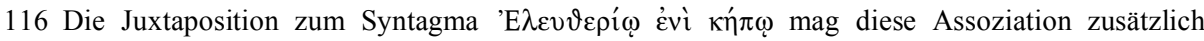
erleichtert haben, denn in antiker Volksetymologie wurde Eileithyia zuweilen mit $\dot{\varepsilon} \lambda \varepsilon v ́ v \varepsilon \rho o \varsigma$ in Verbindung gebracht; vgl. dazu Paschalis (2005b) 111-112.

117 Dies alles muss freilich Spekulation bleiben. Problematisch ist die Assoziation der Artemis mit der Geburtsgöttin, da dies nur eine von vielen ihrer Funktionen und auch keineswegs ihre wichtigste ist. Hinzu kommt, dass Artemis in den homerischen Epen, an welche sich Quintus ja anlehnt, als Geburtsgöttin noch nicht vorkommt; vgl. Schreiber (1884-1890) 571.

118 Hopkinson (1994a) 106: „The significance of lines 311-12 is lost; but the description of the mountain as neither too high nor too low is clearly programmatic, implying that the poem is

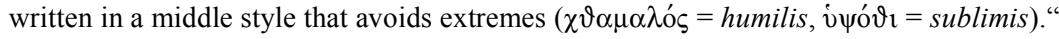

119 Hopkinson (1994a) 106-107: „Neither sublime nor pedestrian, Quintus bases his style on that of Homer while avoiding controversial words and metrical irregularities. He adopts and adapts Homeric epithets and formulas to produce modest innovation within traditional parameters. [...] He parades some, but non excessive, learning; and his gods, though similar in many ways to those of Homer, act with greater decorum. In these as in other respects his motto is $\mu \eta \delta \dot{\varepsilon v}$ örov."

$120 \mathrm{Zu}$ den Stilarten und ihrer Terminologie vgl. Lausberg ( $\left.{ }^{3} 1990\right)$; Calboli (1998).

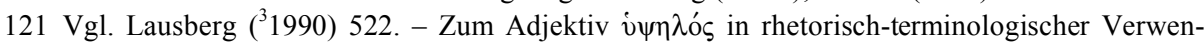

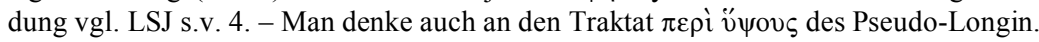

122 Der Gedanke einer Lehnübersetzung des lateinischen humilis scheint mir verlockend.

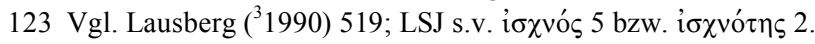


massvollen, undogmatischen Standpunkt anzeigt - offenbar sieht er die drei Stilarten nicht als abgeschlossene Bereiche an, sondern als Eckpunkte einer ganzen Skala von möglichen Ausdrucksweisen, auf welcher er sich eben weder am untersten noch am obersten Ende bewegen will. ${ }^{124}$ Drittens dürfte das von James vorgebrachte Gegenargument, die Gattung Epos gehöre traditionell dem genus grande an und eine poetologische Lesart von Q.S. 12.313 sei deshalb abzulehnen, ${ }^{125}$ eigentlich genau das Gegenteil beweisen: Gerade weil Quintus offenbar von der gängigen Zuordnung des Epos zur Stilhöhe des genus grande abweichen will, muss er die Angelegenheit thematisieren. Dies wiederum würde

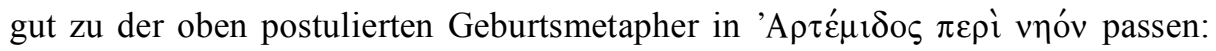
Artemis unterstützt in ihrer Funktion als Geburtshelferin Quintus bei der Kreation einer neuen Art von Epos. Man sollte also nicht eine metaphorisch-poetologische Interpretation der Stelle ablehnen, weil eine solche mit den gängigen Vorstellungen der antiken Rhetorik (angeblich) nicht kompatibel ist, sondern man sollte sich vielmehr fragen, was für mögliche Konsequenzen sich aus einer solchen Inkompatibilität ergeben. Es liesse sich beispielsweise ins Feld führen, dass die homerischen Epen in der Antike die Schullektüre schlechthin und somit jedem, der einigermassen gebildet war, bekannt waren. ${ }^{126}$ Homer zu kennen, war - trotz allem Respekt, den man ihm als Begründer der griechischen Dichtkunst selbstverständlich entgegenbrachte - kein Zeichen von überragender Bildung, sondern von Allgemeinwissen. Entsprechend wurde das Abfassen eines homerisierenden Epos zwar einerseits wohl als sehr ehrgeiziges Unterfangen angesehen, andererseits aber muss sich Quintus bewusst gewesen sein, dass er mit seinen Posthomerica nicht in die allerhöchsten Gefilde der Dichtkunst und der aemulatio vordrang. Beidem trägt er mit seiner Aussage in Vers 313 Rechnung: Nicht nur positioniert er sein Werk an einem passenden Platz im zeitgenössischen Literaturund Bildungsbetrieb, sondern er variiert auch einen weiteren rhetorischliterarischen locus classicus, den Bescheidenheitstopos, welcher hauptsächlich in der spätantiken (christlichen und heidnischen) Literatur verbreitet war. ${ }^{127}$ Mit der

124 Man war sich in der antiken Rhetoriktheorie durchaus bewusst, dass die drei Stilarten keine hermetisch abgeriegelten Bereiche darstellen; vgl. Quint. Inst. 12.10.67: ac sic prope innumerabiles species reperiuntur, quae utique aliquo momento inter se differant. „Und so lassen sich beinahe unzählige 〈Stil-〉Gattungen finden, welche sich, zumindest in einem gewissen Punkt, voneinander unterscheiden.“ Vgl. auch Lausberg $\left({ }^{3} 1990\right)$ 524: „Die drei genera stellen nur eine Auswahl aus den Möglichkeiten der tatsächlich notwendigen Redeweisen dar [...] In der Praxis löst sich das Dreiersystem in eine grosse Zahl von Varianten auf $[\ldots]^{\text {“ }}$

125 James (2004) XVIII: ,[T] he point of such a claim with reference to his epic is not at all obvious. Heroic poetry that seeks to be morally edifying belongs to the upper end of the poetic spectrum."

126 Vgl. Morgan (1998) 105-111.

127 Vgl. Hagenbichler (1992). - Ein epischer Bescheidenheitstopos findet sich beispielsweise in der Vorrede zum christlichen Epos Iohannis des Corippus (5. Jh. n. Chr.), Coripp. praef. 15-16: 


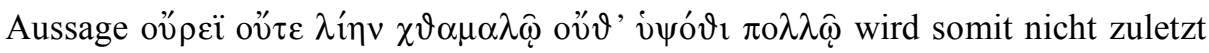
der etwas gar hochgegriffene Anspruch, einen zweitsophistischen Homer-HesiodKallimachos in Personalunion zu verkörpern, ein Stück weit wieder auf ein vertretbares Niveau gedrosselt. Doch indem sich Quintus dergestalt zurücknimmt, bekräftigt er seinen Anspruch, ein Homerus novus zu sein, implizit gleich noch einmal, da er eben entgegen der gängigen Stiltheorie ein Epos (ungefähr) im mittleren Stil zu schreiben beliebt. Es liegt hier also ein ähnlicher Effekt vor, wie wir ihn schon im Zusammenhang mit den Eingangsversen Q.S. 1.1-17 festgestellt haben, nämlich, dass es Quintus gelingt, in einer einzigen Aussage zwei eigentlich gegenteilige Inhalte bzw. Implikationen miteinander zu kombinieren. ${ }^{128}$

In einem gleichen Kontext ist denn wohl auch das allerletzte ,Puzzleteil', die topographische Angabe 'E $\lambda \varepsilon v \vartheta \varepsilon \rho i ́ \omega$ évi $\kappa \dot{n} \pi \omega$ in Q.S. 12.312, zu verstehen: Wenn wir den ganzen Rest der Verse 310-313 poetologisch lesen und in Vers 313 die Aussage des Dichters vernehmen, sein Epos entgegen gängiger Stildefinitionen nicht im genus grande, sondern ,nur' im genus mixtum anzusiedeln, so werden wir den „Garten der Freiheit“" unschwer als ,künstlerische / dichterische Freiheit' auffassen können. ${ }^{129}$

\section{Zusammenfassung, Konsequenzen, Ausblick}

Alles in allem lassen sowohl Quintus' Verzicht auf ein Initialproömium zu Beginn seines Epos als auch sein Binnenproömium vor dem Katalog der Helden, die das hölzerne Pferd besteigen, ein gezieltes künstlerisches Wollen erkennen, welches, allgemein gesprochen, in einer kreativen Auseinandersetzung mit dem episch-homerischen Vorbild im Sinne einer imitatio cum variatione bzw. einer

Aeneam superat melior virtute Iohannes, / sed non Vergilio carmina digna cano. „Den Aeneas übertrifft 〈zwar〉 der an Tugend bessere Johannes, aber ich singe kein dem Vergil würdiges Gedicht."

128 Als Letztes sei noch auf eine Wortoberflächenparallele verwiesen: Mit Q.S. 12.313 vgl. Arat.

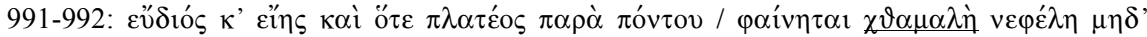
juóvı кúp̣.. „Gutes Wetter wird dir wohl auch beschieden sein, wenn vom flachen Meer her eine tiefliegende Wolke erscheint und sich nicht in der Höhe befindet." Wegen der Verschiedenheit von Inhalt und Kontext lässt sich eine sprachliche Abhängigkeit des Quintus von Arat jedoch nicht beweisen.

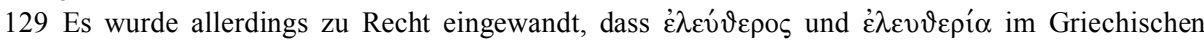
sonst nicht in dieser Bedeutung vorkommen. Herr Prof. Dr. Walter Burkert verweist auf den Topos des Gartens als Ort der Erleuchtung; möglicherweise ist also der „Garten der Freiheit“ stärker im Zusammenhang mit Quintus' Dichterweihe zu sehen. - Martin Wests Konjektur von

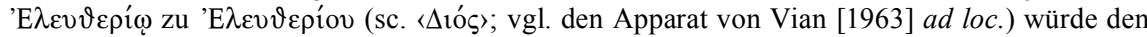
Sinn der Stelle tiefgreifend verändern (,im Garten des Zeus Eleutherios“ statt „im Garten der Freiheit“); zu fragen wäre dann jedoch, wie wir die Präsenz des Zeus in Quintus' Musenanruf zu deuten haben. 
aemulatio besteht. Die Botschaft, ein Epos in Anlehnung an das homerische Vorbild und in gleichzeitiger Absetzung davon bzw. in kritischer Auseinandersetzung damit schreiben zu wollen, wird mittels einer formalen Leerstelle - des Fehlens eines Initialproömiums - vermittelt, was keinesfalls als Ausdruck mangelnder Originalität oder reinen Epigonentums missverstanden werden darf, und im Binnenproömium im 12. Buch erneut aufgegriffen. Daselbst geriert sich Quintus auf einer intertextuell-metapoetischen Ebene als Homerus novus, als Homer-Hesiod-Kallimachos in Personalunion, wobei er diesen hoch gegriffenen Anspruch im letzten Vers durch die Aussage, sein Epos nur im mittleren Stilbereich ansiedeln zu wollen, ein Stück weit wieder zurücknimmt. Vor dem Hintergrund all dessen scheint es naheliegend, wenn nicht beinahe zwingend, die

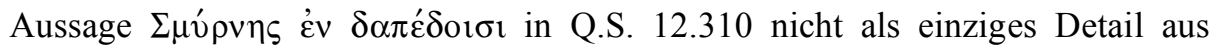
einem ganzen zusammengehörigen Komplex autobiographisch, sondern ebenfalls primär topisch-poetologisch zu lesen: dies in erster Linie aufgrund einer verbreiteten antiken Tradition, dergemäss Smyrna die Geburtsstadt Homers war, ferner jedoch auch im Kontext der Zweiten Sophistik, da Smyrna ein wichtiges Zentrum dieser Strömung war, wodurch sich Quintus auch in den weiteren Zusammenhang seines eigenen kulturellen und intellektuellen Umfelds einschreibt. Freilich muss man einräumen, dass sich die Richtigkeit einer autobiographischen Deutung nicht nur nicht abschliessend beweisen, sondern auch nicht eindeutig widerlegen lässt. Plausibel dürfte sie jedoch nicht sein, und es ist anzunehmen, dass - bei aller gebotenen Vorsicht hinsichtlich solcher Mutmassungen - Quintus eine konkretautobiographische Lesart nicht (oder zumindest nicht primär) intendiert hat.

Als Konsequenz aus alledem ergibt sich, dass Spekulationen etwa hinsichtlich der chronologischen Einordnung der Posthomerica, die sich auf die Lokalisierung unseres Dichters in Smyrna stützen, ${ }^{130}$ oder etwa gar hinsichtlich Quintus' Biographie selber ${ }^{131}$ auf $\mathrm{zu}$ unsicheren Prämissen beruhen und somit methodisch nicht zulässig sind. So müssen wir uns fürderhin damit abfinden, dass wir über Person und Vita des Verfassers der Posthomerica, dessen Heimat man seit Tzetzes aufgrund einer biographistischen Lesart von Q.S. 12.310 in Smyrna ansetzen zu können glaubte, nichts Gesichertes wissen und nur den Namen Kóïv $\tau$ o erst aus byzantinischer Überlieferung kennen.

130 Vgl. etwa Cantilena (2001) 54-55, der glaubt, um ca. 250 n. Chr. einen terminus ante quem für die Abfassung der Posthomerica ansetzen zu können, da um diese Zeit eine Verlagerung der kulturellen Zentren aus dem Bereich Anatoliens nach Konstantinopel, Antiocheia und Alexandreia stattfand und er annimmt, dass Quintus danach nicht mehr hätte erfolgreich in Smyrna literarisch wirken können.

131 Vgl. etwa Appel (1994c) 12-13, oder auch die von Rhodomann (1604) in seiner praefatio erwogene Mutmassung, Quintus könnte ein ,Schulleiter` in Smyrna gewesen sein und mit den $\mu \hat{\eta} \lambda \alpha$ (Q.S. 12.310) habe er metaphorisch seine discipuli gemeint. 
Doch wollen wir nicht mit einer negativen Bilanz enden. Können wir aus den angestrengten Überlegungen und Analysen auch einen Nutzen für das Verständnis des Gesamtwerks ziehen? Einerseits ist hier freilich die hohe künstlerische Qualität der Posthomerica zu nennen, welche aus den subtilen intertextuellen Anspielungen und Verweisen des Binnenproömiums spricht und wodurch wir eingeladen, ja aufgefordert sind, das Gesamtepos als Kunstwerk eines in nachgerade alexandrinischer Manier dichtenden poeta doctus zu lesen und zu geniessen. Andererseits jedoch stellt sich auch die Frage nach dem intendierten Publikum: Was für ein Rezipient mag sich von einer derart anspruchsvollen und anspielungsreichen Intertextualität angesprochen gefühlt und diese genossen, und wie dürfte demgegenüber ein weniger gebildeter Leser (oder Hörer) das Werk wahrgenommen und verstanden haben? Rezente Forschungen zum Publikum der zweitsophistischen Konzertrhetorik und der damit verbundenen Frage nach der soziokulturellen Bedeutung des daselbst zur Schau getragenen Bildungsguts haben gezeigt, dass wir die Zweite Sophistik als ein Phänomen kultureller Selbstdefinition in einem doppelten - d.h. einem weiteren und einem engeren - Sinne $\mathrm{zu}$ verstehen haben. ${ }^{132}$ Einerseits erfährt die griechische Sprach- und

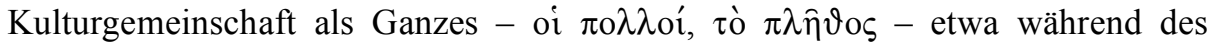
öffentlichen Auftritts eines Sophisten ein Gefühl panhellenischer Identität, wodurch sie sich vom Rest der nichtgriechischen Welt absetzt und sich dieser überlegen fühlt. ${ }^{133}$ Andererseits und gleichzeitig jedoch setzt sich innerhalb dieser Gesamtgemeinschaft eine quantitativ kleine intellektuelle Elite - die Gruppe der $\pi \varepsilon \pi \alpha \_\delta \varepsilon v \mu \varepsilon^{\prime} v o r$-, die sich als Trägerin, Vermittlerin und Bewahrerin ebendieser Identität versteht, auf einem höheren Niveau von der Masse ab, was hinwiederum in deren eigenem Kreis zu einer spezifisch elitären Identitätsstiftung führt. Dieses Modell lässt sich nun, wie mir scheint, auf die mögliche zeitgenössische Rezeption der Posthomerica gewinnbringend übertragen: Jeder leidlich gebildete Grieche des 2. oder 3. Jhs. n. Chr. wird nicht bloss die Figuren und Ereignisse des Trojanischen Krieges gekannt haben, sondern er dürfte auch in der Lage gewesen sein, die homerische Hexametersprache als solche zu erkennen und dem Vortrag eines Ilias-Gesanges zumindest in den groben Zügen zu folgen. Wir können uns also durchaus vorstellen, wie eine Rezitation auch der Posthomerica von einem breiten zeitgenössischen Publikum verstanden und genossen und somit als identitätsstiftend im erstgenannten weiteren Sinne empfunden worden sein mag. Ein solches Publikum wird die in den vorausgegangenen Kapiteln aufgezeigten intertextuellen Anspielungen und Verweise kaum bemerkt, doch etwa an der

132 Vgl. dazu v.a. Schmitz (1997), bes. 160-196, und Korenjak (2000), bes. 41-65.

133 Dies ist allerdings nicht zwingend in einem politischen, d.h. antirömischen, oder eskapistischen, d.h. der Politik und Alltagsrealität entsagenden Sinne zu verstehen; vgl. Schmitz (2000) 178 (mit weiterführender Literatur). 
mitreissenden Schilderung von Penthesileias Angriff gegen die Achaier und Achilleus sowie am archaischen Klang der homerisierenden Sprache nichtsdestoweniger seine Freude gehabt und sich also in jenem Moment als zusammengehörige Einheit mit einem gemeinsamen kulturellen und sprachlichen Erbe gefühlt haben. ${ }^{134}$ Die sachverständigen $\pi \varepsilon \pi \alpha 1 \delta \varepsilon v \mu \varepsilon ́ v o r$ jedoch sind es, welche darüber hinaus die Feinheiten, Spezialitäten und Anspielungen in Quintus' Sprache verstanden und geschätzt haben und sich so in ihrer Identität in dem zweitgenannten engeren Sinne innerhalb ihres eigenen elitären Kreises desgleichen bestätigt fühlten. Ja, wir könnten vielleicht sogar so weit gehen uns vorzustellen, wie die ,breite Masse' bei einer (von uns imaginierten) Posthomerica-Rezitation die neuen Texte für ,echt homerisch“ gehalten haben mag, während die $\pi \varepsilon \pi \alpha_{1-}$ $\delta \varepsilon v \mu \varepsilon ́ v o r$ die zahlreich vorhandenen, jedoch subtilen Unterschiede - beispielsweise im Sprachgebrauch (etwa bezüglich unhomerischer Wörter, Formen oder Formeln) oder hinsichtlich abweichender Sagenversionen im Kleinen ${ }^{135}$ - bemerkten und sich so hinwiederum der Schicht der gutgläubigen weniger Gebildeten überlegen fühlen konnten. ${ }^{136}$

Der gegenwärtige Forschungsstand zu den Posthomerica sowie v.a. die Quellenlage, d.h. das Fehlen zeitgenössischer Rezeptionszeugnisse, erlauben leider keine spezifischeren Aussagen zu diesem ganzen Themenkomplex. Doch während wir bezüglich der Identität unseres Dichters sozusagen vollständig im Dunkeln tappen, so lässt sich über die Identität seines Texts als Medium kultureller Identitätsstiftung zumindest erfolgreich spekulieren.

134 Vgl. Schmitz (1997) 167: „Was insbesondere die Improvisationen der Sophisten betrifft, so muß man davon ausgehen, daß die Häufigkeit dieser Auftritte, verbunden mit dem recht eingeschränkten Kreis der behandelten Themen, jedem Bürger, der im Ambiente einer durch griechische Kultur geprägten $\pi$ ó $\lambda 1 \varsigma$ aufwuchs, durch die unablässige Wiederholung zumindest einen gewissen Grundvorrat an Wissen vermittelte, auf den jeder Redner zählen konnte. Gewiß war das Publikum in seiner Gesamtheit nicht in der Lage zu beurteilen, inwieweit ein Redner Fehler in der Beherrschung der attizistischen Kunstsprache machte, doch war es immerhin imstande, diese Sprache zu verstehen und ihren feierlichen, archaischen Klang zu spüren. Die besonders geliebten historischen $\mu \varepsilon \lambda \varepsilon \varepsilon^{\prime} \alpha \iota \iota$ umfaßten einen so geringen Zeitraum der griechischen Geschichte (und auch aus diesem nur wenige markante Ereignisse), daß ein Zuhörer, der eine Reihe von Auftritten miterlebt hatte, mit ihnen vertraut war [...]“

135 Vgl. dazu den Beitrag von Knut Usener in diesem Band.

136 In diesem Zusammenhang wäre wiederum daran zu denken, in welch hohem Masse Homer und die homerischen Gedichte gerade in der Zweiten Sophistik als identitätsstiftend in einem panhellenischen Sinne empfunden wurden; vgl. z.B. Aelius Aristides, der Homer als ó кoเvò $\tau \hat{\omega} v$

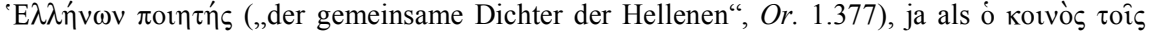

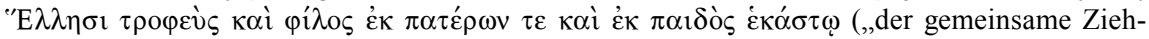
vater für die Hellenen, ein Freund für ein jeden seit alters her und von Kindesbeinen an“, Or. 17.15) bezeichnet. 